\title{
A Hybrid N-body-Coagulation Code for Planet Formation
}

\author{
Benjamin C. Bromley \\ Department of Physics, University of Utah, 201 JFB, Salt Lake City, UT 84112 \\ e-mail:bromley@physics .utah.edu \\ Scott J. Kenyon \\ Smithsonian Astrophysical Observatory, 60 Garden Street, Cambridge, MA 02138 \\ e-mail: skenyon@cfa.harvard.edu
}

\begin{abstract}
We describe a hybrid algorithm to calculate the formation of planets from an initial ensemble of planetesimals. The algorithm uses a coagulation code to treat the growth of planetesimals into oligarchs and explicit $N$-body calculations to follow the evolution of oligarchs into planets. To validate the $N$-body portion of the algorithm, we use a battery of tests in planetary dynamics. Several complete calculations of terrestrial planet formation with the hybrid code yield good agreement with previously published calculations. These results demonstrate that the hybrid code provides an accurate treatment of the evolution of planetesimals into planets.
\end{abstract}

Subject headings: planetary systems - solar system: formation - stars: formation circumstellar matter

\section{INTRODUCTION}

Rocky planet formation begins as gas and dust around a young star settle into a thin disk. The emergence of planets within this disk is the result of three phases of evolution (Safronov 1969; Weidenschilling 1980; Hayashi 1981; Wetherill \& Stewart 1993; Ida \& Makino 1993; Kokubo \& Ida 1996). Initially, coagulation of dust particles causes a stochastic but steady growth in particle mass. The few largest bodies, or planetesimals, accumulate mass most quickly, and experience a runaway growth phase. As the largest objects clear out the smaller ones, the planetesimal growth is oligarchic, where the largest objects - "oligarchs" - become isolated from their neighbors and grow roughly at the same rate. In a final phase of chaotic growth, mergers of oligarchs lead to the formation of a few terrestrial planets around Sun-like stars well within 100 Myr (e.g., Weidenschilling et al. 1997; Chambers 2001; Kokubo \& Ida 2002; Kokubo et al. 2006; Kominami \& Ida 2002). 
This scenario is bolstered by recent observations. Most, if not all, young late-type stars have a disk of gas and dust (Backman \& Paresce 1993; Beckwith 1999; Lada 1999). At least some of these disks will form large planets, similar to those detected in radial velocity studies (e.g., Marcy \& Butler 2000). The persistence of dusty disks around older stars suggests that smaller planets form as well. Dust should be ejected by radiation pressure, but on-going formation of rocky planets can drive a collisional cascade which continually produces dust from a reservoir of small planetesimals (Kenyon \& Bromley 2001, 2002a, 2002b). Stars with such debris disks include $\beta$ Pic (Kalas et al. 2000; Wahhaj et al. 2003), $\epsilon$ Eri (Greaves et al. 1998), HR 4796A (Jayawardhana et al. 1998; Koerner et al. 1998) and Vega (e.g., Koerner, Sargent \& Ostroff 2001).

Interpretations of observed dusty debris disks, and our understanding of planet formation in general, rely heavily on numerical calculations. Two types of tools, statistical solvers and $N$-body codes, provide complementary information about planetary disk evolution. The effectiveness of each tool depends on $N$, the number of particles they can track. Statistical methods like Safronov's (1969) particle-in-cell formalism work well when the particles are numerous and have small mass (Kokubo \& Ida 1996). Current $N$-body codes are unable to follow planetary growth in this regime. When the mass of individual objects gets large, binary interactions become important. Orbit evaluations then require direct $N$-body calculations, not the ensemble averages of particlein-cell approaches. Our experience with coagulation codes (Kenyon \& Luu 1998, 1999; Kenyon \& Bromley 2001, 2002b, 2003) suggests that we can accurately evolve objects with masses below $10^{25}-10^{26} \mathrm{~g}$ using the statistical approach. However, an $N$-body code must track particles of heavier mass (see also Kokubo \& Ida 1996, 2002).

Massive planetesimals requiring direct $N$-body evolution are relatively rare in typical planet formation models. This situation is fortunate because $N$-body codes can not accurately track large numbers of particles for long periods of time. Simulations performed by Chambers (2001) and some runs reported here involve $N \sim O(100)$ particles integrated over 100 Myr. Ida, Kokubo \& Kominami (2003) ran larger simulations with $N=10,000$, but only for a $\sim 0.5$ Myr time period and only with specialized hardware for gravitational force calculations.

The complementary limitations of $N$-body algorithms and coagulation codes call for an integration of both methods into a single "hybrid code" (Jewell \& Alexander 1996; Weidenschilling et al. 1997) Here we describe an algorithm that includes both a statistical component and a direct $N$ body part. Our goal is to run self-consistent simulations of planet formation, tracking objects from micron-sized dust grains to Jupiter mass planets. The coagulation code, described briefly below in $\S 2$, accounts for gas, dust, and a swarm of lower mass planetesimals. Our $N$-body algorithm ( 33 ), is invoked only when needed to evolve the largest planetesimals. In $\S 4$ we discuss the hybrid code itself, and focus on how the $N$-body and coagulation parts interact. We provide tests of this code in $\S 5$ and give preliminary results related to the problem of terrestrial planet formation. 


\section{THE COAGULATION CODE}

The coagulation part of our hybrid code tracks dust and planetesimals in multiple annuli around the central star. Kenyon \& Luu (1998, 1999) and Kenyon \& Bromley (2001, 2002b, 2004a) describe the algorithms and include a complete set of references. Here we briefly review this statistical algorithm.

The physical processes which we simulate include coagulation, fragmentation, gas drag, PoyntingRobertson drag, and radiation pressure. We discretize the continuous distribution of particle masses into mass batches, assigning an integral number of particles to each batch. To allow better resolution of the mass spectrum for particles evolving rapidly, adjacent batches differ in mass by a mass-dependent factor. We dynamically adjust the number of mass batches, as needed. Our spatial domain is cylindrical and is divided into a set of concentric annuli about the central star. The annuli may be set so that either they have equal width, or their boundaries are equally spaced in log-radius.

Masses and particle numbers evolve according to the coagulation equations which include the effects of collisions, Poynting-Robertson drag, and interaction with the gas disk. Our collisions rates come from geometric cross sections of particles, augmented by a gravitational focusing factor for larger planetesimals. Collision outcomes depend on the planetesimal tensile strength, gravitational binding energy, and relative velocities. Depending on these parameters, collisions result in mergers, fragmentation, and dust production.

We follow particle velocities statistically for each mass batch in each annulus, tracking vertical and horizontal velocity dispersions relative to Keplerian orbits in the central plane of the disk. These dispersions evolve under the influence of gas drag, Poynting-Robertson drag, viscous stirring and dynamical friction, according to a set of Fokker-Planck equations (Hornung, Pellat \& Barge 1985; Wetherill \& Stewart 1993; Stewart \& Ida 2000; Ohtsuki, Stewart, \& Ida 2002).

To solve the coagulation and Fokker-Planck equations, we use a fourth-order Runge-Kutta method. The algorithm strictly conserves mass. When objects shift from one annulus to another, velocity dispersions are updated to conserve kinetic energy explicitly. At the inner and outer boundaries of the spatial domain, the mass batches reflect a steady, radial flow in the debris disk. The code does not enforce angular momentum conservation, nonetheless, in test runs of $10^{6}$ timesteps, angular momentum is conserved to better than $1 \%$.

The coagulation code evolves the swarm of $O\left(10^{21}\right)$ planetesimals accurately until the largest objects reach roughly the mass of Pluto. Because these massive objects are rare, the statistical

model provides a poor estimate of their behavior. Although the coagulation code can still follow the evolution of the swarm, the orbits of the largest objects require explicit calculations using an $N$-body code, which we describe in the next section. 


\section{THE $N$-BODY CODE}

Planetary $N$-body codes must be fast and accurate. Suitable solvers for the equations of motion are numerous, with published descriptions of symplectic integrators (Wisdom \& Holman 1991; Kinoshita, Yoshida \& Nakai 1991; Saha \& Tremaine 1992), and more general time-symmetric integrators (e.g., Quinlan \& Tremaine 1990, Hut, Makino \& McMillan 1995). Some integrators (Vasilev 1982; Wisdom \& Holman 1991; Ida \& Makino 1992; Fukushima 1996; Shefer 2002) speed up the calculations by tracking only deviations from a purely Keplerian orbit about the central star, following the proposal of Encke (1852). Other improvements include adaptive timestepping, allowing integrators to take large timesteps when forces are small, and to expend more computational resources only when particles experience strong forces (Aarseth 1985; McMillan \& Aarseth 1993; Skeel \& Biesiadecki 1994; Kokubo, Yoshinaga \& Makino 1997; Duncan, Levison \& Lee 1998).

Our algorithm is based on the Encke method. We work with non-inertial, Keplerian frames about the central star and integrate the equations of motion in rectilinear coordinates defined relative to the Keplerian frames. These coordinates have a fixed orientation, aligned with the inertial frame of the central star. We use an adaptive block timestepping scheme (e.g., McMillan \& Aarseth 1993) and a sixth- or eighth-order accurate integrator based on Richardson extrapolation. We give details of the integrator below. For now we briefly outline the timestepping scheme.

At the beginning of a timestep, each particle's accelerated reference frame is initially set to its instantaneous Keplerian orbit. If particles are in tight groups, their reference frames can be set to the Keplerian orbit of their mutual center of mass. A friends-of-friends algorithm (Huchra \& Geller 1982, Geller \& Huchra 1983) finds any such groups with a linking parameter based on the Hill radii of the particles. We then integrate the equations of motion in terms of spatial variables in the accelerated reference frames over a single timestep of length $\Delta t$. Next we calculate higher resolution orbits by dividing the timestep into $m$ equal substeps. In practice, $m=3$ seems to work best. A comparison between the lower and higher resolution orbits produces a check of integrator convergence. We have implemented several criteria for convergence, but have found that an individual particle's total energy, taken as a fraction $\epsilon$, of its Keplerian orbital energy, gives an effective comparison under most circumstances. If a particle's orbit has not converged, we simply repeat the process at even higher time resolution.

The strategy of a block timestepping scheme is to track individual orbits and interpolate the converged orbits if needed to evaluate forces on particles whose orbits have not converged. This procedure reduces the computational load of force calculations if the number of particles is large $(N \gg 10)$. For a smaller number of particles we prefer to integrate all orbits until every orbit has converged. Energy conservation is better in this case and there is no significant penalty in computational load.

The time integrator is an ordinary differential equation (ODE) solver based on Richardson extrapolation. We start with a low-resolution estimate of the particle orbits over a time interval

$\Delta t$ using a leapfrog intergrator. Specifically, the position $\vec{x}$ and velocity $\vec{v}$ of a particle at the end 
of a time-forward step in the leapfrog case is

$$
\begin{array}{ll}
\vec{v}(t+\Delta t / 2) & =\vec{v}(t)+\Delta t / 2 \vec{a}(t) \\
\vec{x}(t+\Delta t) & =\vec{x}(t)+\Delta t \vec{v}(t+\Delta t / 2) \\
\vec{v}(t+\Delta t) & =\vec{v}(t+\Delta t / 2)+\Delta t / 2 \vec{a}(t+\Delta t)
\end{array}
$$

where $\vec{a}$ is the acceleration, which in our case depends only on position. We label the position at the end of this single leapfrog timestep as $\vec{x}_{0}$, and note that it contains errors of the order of $\Delta t^{3}$. Next we divide the time interval into two equal parts and take two successive leapfrog steps to derive a better resolved orbital position, $\vec{x}_{1}$. We can further subdivide the time interval to obtain a position $\vec{x}_{i}$, derived from $2^{i}$ successive leapfrog timesteps. Our final position at the end of the time interval $\Delta t$ is a linear combination of these results,

$$
\vec{x}(t+\Delta t)=\sum_{i=0}^{m} c_{i} \vec{x}_{i}+O\left(\Delta t^{2 m+1}\right) .
$$

Fourth-, sixth-, and eighth-order methods have $m=1,2$ and 3, respectively and coefficients

$$
\begin{aligned}
\left(c_{0}, c_{1}\right) & =(4 / 3,-1 / 3) & & 4^{\text {th }}-\text { order } \\
\left(c_{0}, c_{1}, c_{2}\right) & =(1 / 45,-4 / 9,64 / 45) & & 6^{\text {th }}-\text { order } \\
\left(c_{0}, c_{1}, c_{2}, c_{3}\right) & =(-1 / 2835,4 / 135,-64 / 135,4096 / 2835) & & 8^{\text {th }} \text { order }
\end{aligned}
$$

We calculate the gravitational forces between particles directly. At the lowest resolution timesteps, we perform the $\mathrm{O}\left(N^{2}\right)$ force evaluations. However, if we use the block timestepping scheme then only a few particles depend on $\mathrm{O}(N)$ interparticle forces at high temporal resolution. To simulate a larger number of bodies, we have an $\mathrm{O}(N \log N)$ Barnes \& Hut (1986) treecode, as described in Barton, Bromley \& Geller (1999). Preliminary tests suggest that this code becomes competitive with a direct method only when $N$ is greater than $\mathrm{O}\left(10^{3}\right)$. An alternative would be to use specialized hardware (e.g., Hut \& Makino 1999). Here, our particle numbers are small and we work exclusively with the direct force solver.

For realistic planet formation models, an $N$-body algorithm must identify merger events. We check for mergers in a fast way, by assuming that any merger event which occurs during a single timestep can involve only two particles. For each particle we save an index number corresponding to some other particle which came the closest during the force calculations. This indexing reduces the number of calculations from $O\left(N^{2}\right)$ to $O(N)$. For each pair of bodies, with indices $i$ and $j$, we check the relative radial velocity using the quantity $\vec{x}_{i j} \cdot \vec{v}_{i j}$, where the vectors are relative position and velocity respectively. If the relative radial velocity is positive at the beginning of a timestep, then we assume no merger takes place. Otherwise, we find the minimum separation of the pair during the timestep, interpolating if necessary. Once we have the minimum pair separation $s_{i j}$ and relative speed $v_{i j}$, a merger event is identified if

$$
s_{i j} \leq \min \left[R_{i}+R_{j}, \frac{1}{v_{i j}}\left(v_{E, i} R_{i}+v_{E, j} R_{j}\right)\right]
$$


where $v_{E, i}$ is the escape velocity at distance $s_{i j}$ from the $i^{\text {th }}$ particle, and $R_{i}$ is the particle's physical radius. Thus the merger cross-section is at least the physical cross-section, and is larger if the relative velocity is small compared to the escape velocity of either particle.

\section{THE HYBRID CODE}

With our coagulation code and $N$-body algorithm, we track both the numerous low-mass planetesimals and the relatively rare high-mass bodies in a planetary disk. The interactions between these two populations generally cause the larger bodies to circularize in their orbits, while the smaller bodies tend to get gravitationally stirred, as reflected in an overall increase in eccentricity and inclination. Here we describe how the coagulation and $N$-body components work together to simulate these effects.

The multiannulus coagulation code evolves all low-mass bodies. The model grid contains $N$ concentric annuli with widths $\delta a_{j}$ centered at heliocentric distances $a_{j}$. Each annulus contains $n\left(m_{j l}, t\right)$ objects of mass $m_{j l}$ with orbital eccentricity $e_{j l}(t)$ and inclination $i_{j l}(t)$ in $M$ mass batches. When an object in the coagulation code reaches a preset mass, it is 'promoted' into the $N$-body code. To set the initial orbit for this object, we use the three coagulation coordinates, $a, e$, and $i$, and select random values for the longitude of periastron and the argument of perihelion. Because the annuli have finite width $\delta a_{j}$, we set the semimajor axis of the promoted object to $a_{p}=$ $a_{j}+(0.5-x) \delta a_{j}$, where $x$ is a random number between 0 and 1 . When two or more objects within an annulus are promoted to the $N$-body code during the same timestep, we restrict the choices of the orbital elements to minimize orbital interactions between the newly promoted $N$-bodies.

The coagulation code also determines the simulation timestep. This interval, $\Delta T$, is generally larger than the lowest resolution timestep of the $N$-body code, so we take multiple low-resolution substeps, $\Delta t$, within that interval. The length of a substep is set by the orbital speed of the fastest moving body; for nearly circular orbits this limit is roughly $1 / 60^{\text {th }}$ of the orbital period.

To calculate the effects of the swarm of low-mass bodies on the $N$-bodies, we use particle-ina-box estimates. For an N-body with index $j$, mass $m_{j}$, eccentricity $e_{j}$, inclination $i_{j}$, horizontal velocity $h_{j}$, and vertical velocity $v_{j}$, we derive the time-evolution of the orbital eccentricity and inclination from the Fokker-Planck formulae for the derivatives of the horizontal and vertical velocities:

$$
\begin{aligned}
& \frac{d h_{v s, j, h i g h}^{2}}{d t}=\sum_{k=1}^{N} \sum_{m=1}^{M} f_{j k} C\left(h_{j}^{2}+h_{k m}^{2}\right) m_{k m} J_{e}\left(\beta_{k m}\right) \\
& \frac{d v_{v s, j, h i g h}^{2}}{d t}=\sum_{k=1}^{N} \sum_{m=1}^{M} f_{j k} C\left(v_{j}^{2}+v_{k m}^{2}\right) m_{k m} J_{z}\left(\beta_{k m}\right)
\end{aligned}
$$


for viscous stirring in the high velocity limit and

$$
\begin{aligned}
& \frac{d h_{d f, j, h i g h}^{2}}{d t}=\sum_{k=1}^{N} \sum_{m=1}^{M} 1.4 f_{j k} C\left(m_{k m} h_{k m}^{2}-m_{j} h_{j}^{2}\right) H_{e}\left(\beta_{k m}\right) \\
& \frac{d v_{d f, j, h i g h}^{2}}{d t}=\sum_{k=1}^{k=N} \sum_{m=1}^{M} 1.4 f_{j k} C\left(m_{k m} v_{k m}^{2}-m_{j} v_{j}^{2}\right) H_{z}\left(\beta_{k m}\right)
\end{aligned}
$$

for dynamical friction in the high velocity limit. In these expressions, $\beta_{k m}^{2}=\left(i_{j}^{2}+i_{k m}^{2}\right) /\left(e_{j}^{2}+e_{k m}^{2}\right)$, $C=0.2767 A_{\Lambda} G^{2} \rho_{m} /\left(h_{j}^{2}+h_{k m}^{2}\right)^{3 / 2}$, and the functions $H_{e}, H_{z}, J_{e}$, and $J_{z}$ are definite integrals (Stewart \& Ida 2000; Ohtsuki, Stewart, \& Ida 2002). The subscript $k$ is the annular index while subscript $m$ is the index of a mass batch. The overlap fraction $f_{j k}$ is the fraction of bodies in annulus $k$ that approach within $2.4 R_{H}$ of the $N$-body. We set $\rho_{m}=M_{m} / V_{m}$, where $M_{m}$ is the total mass of bodies with $m_{m}$ in annulus $l$ and $V_{l}$ is the volume of annulus $l$. Following Stewart \& Ida (2000), we also set $A_{\Lambda}=\ln \left(\Lambda^{2}+1\right)$, where

$$
\Lambda=0.1886(h+1.25 v) v / v_{H, j k}^{3}
$$

with $h^{2}=h_{j}^{2}+h_{k m}^{2}, v^{2}=v_{j}^{2}+v_{k m}^{2}, v_{H, j k}=r_{H, j k} v_{K, j k}, r_{H, j k}^{3}=\left(m_{j}+m_{k m}\right) / M_{\odot}$, and $v_{K, j k}=$ $0.5\left(v_{K, j}+v_{K, k}\right)$; the subscript $K$ denotes a Keplerian velocity (see also Kenyon \& Bromley 2002b; Ohtsuki, Stewart, \& Ida 2002).

When the relative velocities of particles approach the Hill velocity, $v_{H, j k}$, we set

$$
\begin{gathered}
C_{1}=\frac{\ln \left(10 \Lambda^{2} /\left\langle e^{2}\right\rangle+1\right)}{10 \Lambda^{2} /\left\langle e^{2}\right\rangle} \\
C_{2}=\frac{\ln \left(10 \Lambda^{2}\left\langle e^{2}\right\rangle^{1 / 2}+1\right)}{10 \Lambda^{2}\left\langle e^{2}\right\rangle^{1 / 2}} \\
C_{3}=\frac{\ln \left(10 \Lambda^{2}+1\right)}{10 \Lambda^{2}}
\end{gathered}
$$

and use

$$
\begin{gathered}
\frac{d h_{v s, j, l o w}^{2}}{d t}=\sum_{k=1}^{k=N} \sum_{l=1}^{l=M} 73 f_{j k} C_{1} r_{H, j k}^{4} \\
\frac{d v_{v s, j, l o w}^{2}}{d t}=\sum_{k=1}^{k=N} \sum_{l=1}^{l=M} f_{j k} C_{2}\left(4\left\langle i^{2}\right\rangle+0.2\left\langle e^{2}\right\rangle^{3 / 2}\left\langle i^{2}\right\rangle^{1 / 2}\right) r_{H, j k}^{4}
\end{gathered}
$$


for viscous stirring in the low velocity limit and

$$
\begin{aligned}
& \frac{d h_{d f, j, l o w}^{2}}{d t}=\sum_{k=1}^{k=N} \sum_{l=1}^{l=M} 10 f_{j k} C_{3}\left\langle e^{2}\right\rangle r_{H, j k}^{4} / h \\
& \frac{d v_{v s, j, l o w}^{2}}{d t}=\sum_{k=1}^{k=N} \sum_{l=1}^{l=M} 10 f_{j k} C_{3}\left\langle i^{2}\right\rangle r_{H, j k}^{4} / h
\end{aligned}
$$

for dynamical friction in the low velocity limit (Ohtsuki, Stewart, \& Ida 2002). In these expressions, $C_{4}=0.3125 \rho_{m} H_{k l}\left(a_{j}+a_{k}\right) V_{K, j k}^{3} /\left(M_{j}+M_{k m}\right)^{2}, H_{j k}$ is the mutual scale height, $\left\langle e^{2}\right\rangle=\left(e_{j}^{2}+\right.$ $\left.e_{k m}^{2}\right) / r_{H, j k}^{2}$, and $\left\langle i^{2}\right\rangle=\left(i_{j}^{2}+i_{k m}^{2}\right) / r_{H, j k}^{2}$. The combined velocity stirring is then

$$
\begin{aligned}
& \frac{d h_{v s, j}^{2}}{d t}=\frac{d h_{v s, j, h i g h}^{2}}{d t}+\frac{d h_{v s, j, l o w}^{2}}{d t} \\
& \frac{d v_{v s, j}^{2}}{d t}=\frac{d v_{v s, j, h i g h}^{2}}{d t}+\frac{d v_{v s, j, l o w}^{2}}{d t}
\end{aligned}
$$

for viscous stirring and

$$
\begin{aligned}
& \frac{d h_{d f, j}^{2}}{d t}=\frac{d h_{d f, j, h i g h}^{2}}{d t}+\frac{d h_{d f, j, l o w}^{2}}{d t} \\
& \frac{d v_{d f, j}^{2}}{d t}=\frac{d v_{d f, j, h i g h}^{2}}{d t}+\frac{d v_{d f, j, l o w}^{2}}{d t}
\end{aligned}
$$

for dynamical friction.

To calculate the accretion rate of planetesimals onto the $N$-bodies, we use the standard coagulation equation:

$$
\frac{d m_{j}}{d t}=\sum_{k=1}^{k=N} \sum_{l=1}^{l=M} A_{j k m} f_{j k} n_{k m} m_{k m}
$$

where $A_{j m k}$ is the normalized cross-section (see Kenyon \& Luu 1999).

To calculate the stirring of planetesimals by $N$-bodies, we calculate the appropriate FokkerPlanck terms for viscous stirring and dynamical friction and add these results to the long distance stirring of Weidenschilling (1989) 


$$
\frac{d h_{l r, k m}^{2}}{d t}=\sum_{j=1}^{j=N_{n}} \frac{G^{2} m_{j}^{2} \Delta a}{V_{K, j k}\left(\delta a^{2}+0.5 H_{j k}^{2}\right)^{2}},
$$

where $N_{n}$ is the number of $N$-bodies and $\Delta a=a_{j}-a_{k}$.

At the end of each coagulation timestep, we pass the stirring and accretion rates for each $N$-body - $d e_{j} / d t, d i_{j} / d t$, and $d m_{j} / d t$ - to the $N$-body code. At the end of every low resolution timestep in the $N$-body code, we modify each particle's orbit and mass to reflect these changes. When possible, we simply redirect the velocity vector so that the eccentricity varies independently of inclination and semimajor axis. The $N$-body functions return an updated particle list to the coagulation code. New orbital positions and masses of the large objects are then reinserted into the coagulation grid. The coagulation calculations proceed in the standard fashion, except that the orbital velocities of the large-mass objects in the grid are not evolved. With a complete circuit from the coagulation code to the $N$-body code to the coagulation code, $N$-bodies influence the evolution of the swarm and the swarm influences the evolution of the $N$-bodies.

\section{TESTS}

We have published elsewhere results on the performance of the coagulation code, as described in $\S 2$. However, the $N$-body and hybrid codes are new, and our purpose here is to validate them. In this section we first test the $N$-body code for stability, dynamic range, accuracy, and merger resolution, mostly following Duncan, Levison \& Lee (1998) in the validation of their SyMBA algorithm. We then test the hybrid code against several simulations of terrestrial planet formation at 1 AU (Weidenschilling et al. 1997; Chambers 2001).

The following subsections are organized according to the type of calculation. We test stability during long term orbit integrations of the major planets and a "scaled outer solar system" where the masses of the major planets are increased by a factor of 50 (Duncan, Levison \& Lee 1998). Then we test dynamic range with two binary planet configurations. The code's accuracy is also established with a test to resolve a critical orbital separation between two planetesimals which determines whether their orbits will cross. In considering mergers, we reproduce the Greenzweig \& Lissauer (1990) results for planetary accretion rates. We also derive the integration accuracy required to track the collision between a massive object and a small projectile on opposing circular orbits at 1 AU. Finally, we simulate terrestrial planet formation, following Weidenschilling et al. (1997), who consider the evolution of km-sized planetesimals, and Chambers (2001), who models the collisional evolution of lunar mass bodies. 


\section{Long-term Orbit Integration}

To evaluate the stability of our adaptive integrator, we evolve the four major planets in orbit about a stationary Sun. Figure 1 shows the behavior of the outer planets' eccentricities over a 10 Myr period. We use two different integrators, a sixth-order accurate symplectic integrator (Yoshida 1990) and our adaptive code, also with a sixth-order accurate ODE solver for comparison. We run both codes at low and high resolution. The run times of the two codes are comparable at each resolution. As illustrated in the figure, the low resolution adaptive code obtains a better measure of eccentricity than its symplectic counterpart. One reason is that the adaptive code is not subject to accumulation of phase errors as in a symplectic algorithm. On the other hand, the adaptive code produces a secular drift in energy over the course of the simulation, whereas the symplectic code does not. The fractional error in total energy at $10 \mathrm{Myr}$ is $10^{-3}$ and $10^{-5}$ for the low and high resolution adaptive code, respectively.

\section{Scaled Outer Solar System}

If the masses of each of the four major planets increase by a factor of 50 , the outer solar system is unstable (see, for example, Duncan \& Lissauer 1998). To verify that our code yields the correct timescale for this instability, we made tests similar to one performed by Duncan, Levison \& Lee (1998). As expected, the semimajor axis of Jupiter's orbit shrinks by a modest fraction of the original semimajor axis, while Saturn is ejected on a timescale of 1,000 years. As Saturn leaves the Solar System, its orbit crosses and excites the orbits of Uranus and Neptune. An increase in the orbital eccentricity of Uranus or Neptune leads to a second ejection, as seen in Figure 2.

\section{Binaries}

Duncan, Levison \& Lee (1998) note that bound binary planets whose center of mass revolves around a star provide a strong test of the ability of an $N$-body integrator to handle a wide range of dynamical timescales. They set up a pair of Jupiter-mass planets with a center of mass on a circular orbit at $1 \mathrm{AU}$. The planets orbit each other with an initial binary semimajor axis of $0.0125 \mathrm{AU}$ and binary eccentricity of 0.6 . Their simulation time was $100 \mathrm{yr}$, covering about 3,200 binary orbits.

We reproduce the Duncan, Levison \& Lee (1998) test. We can limit total energy errors to within a part in 10 million, while keeping the wall time well below a minute on a $1.7 \mathrm{GHz} \mathrm{AMD}$ Athlon processor. Figure 3 illustrates changes in the binary semimajor axis.

The figure also demonstrates a test of our block timestepper. If a small particle is in a tightly bound orbit around a much more massive object, then the position of the massive particle is interpolated in the force calculations for the small body. Our test simulates the orbit of a massless

"spy satellite" on a polar orbit about the Earth. Figure 3 shows that the distance of the satellite 
from the center of the Earth varies by about 100 meters during an orbit. There is no significant drift in its mean altitude over the course of 100 years, corresponding to roughly 600,000 satellite orbits of the Earth.

\section{Planetesimals}

We next test the ability of the $N$-body code to handle the evolution of planetesimals. First, we consider an isolated pair of planetesimals, with masses of $2 \times 10^{26} \mathrm{~g}$. These bodies start on corotating, circular orbits near 1 AU. We specify their orbital separation in terms of their mutual Hill radius, $R_{H}=\left[\left(m_{1}+m_{2}\right) / 3 \mathrm{M}_{\odot}\right]^{1 / 3}$. There is a critical separation, $\Delta a_{\text {crit }}=2 \sqrt{3} R_{H}$, which determines the onset of chaotic behavior: If the planetesimals' initial orbital separation is smaller than $\Delta a_{\text {crit }}$, their orbits will cross, otherwise they will simply scatter to larger orbital separations. Figure 4 shows the evolution of semimajor axes in cases where orbital separations are initially smaller than $\Delta a_{\text {crit }}$ (top panel) and greater than $\Delta a_{\text {crit }}$ (middle panel). As expected, the more closely-spaced pair experiences several orbit crossings while the more distant pair does not. Multiple calculations demonstrate that our code can resolve the critical separation $\Delta a_{\text {crit }}$ to within a few percent.

Figure 4 also shows results for eight planetesimals, each with the same mass as in the preceding case, and initially on circular, Keplerian orbits. The separation between nearest neighbors is $1.05 \Delta a_{\text {crit }}$. The orbit crossings proceed from larger radius inward, as illustrated anecdotally in the figure (see also Weidenschilling et al. 1997; Kenyon \& Bromley 2001).

Next we use the $N$-body code to model gravitational stirring by two $2 \times 10^{26}$ g objects in a uniform disk of 805 lower mass, $2 \times 10^{24}$ g objects (Kokubo \& Ida 1995; Weidenschilling et al. 1997; Kenyon \& Bromley 2001). The disk is centered at $1 \mathrm{AU}$, and is $35 R_{H}$ in annular extent, where $R_{H}$ is the mutual Hill radius between large and small objects. To speed up the code's convergence with this relatively large number of particles, we soften the interaction potential on a scale comparable to the physical radius of the large bodies, assuming their density is $1 \mathrm{~g} \mathrm{~cm}^{-3}$.

Figure 5 illustrates the evolution of the radial profile of $\left(e^{2}+i^{2}\right)^{1 / 2}$ for the case where the two massive bodies are separated by $10 R_{H}$, and are each at an orbital distance of $5 R_{H}$ away from $1 \mathrm{AU}$. The initial eccentricity and inclinations are zero for the two massive objects, and small $\left(i \sim 10^{-5}\right.$, $e \sim 10^{-7}$ ) for the planetesimals. The $N$-body results here compare well with those from the pure coagulation code (Kenyon \& Bromley 2001), except that the $N$-body calculations produce more structure in the profile, particularly an enchanced scattering of the swarm away from the more massive bodies. This difference, a broadening of the profile as compared to the coagulation results, is expected from previous $N$-body calculations (Kokubo \& Ida 1995). 


\section{Mergers}

One test of our merger algorithm is provided by Greenzweig \& Lissauer (1990), who studied gravitational focusing by a single planet in a field of test particles, all orbiting a $1 \mathrm{M}_{\odot}$ star. We consider one of their configurations, a $10^{-6} \mathrm{M}_{\odot}$ planet on a circular orbit at $1 \mathrm{AU}$ and a set of test particles on orbits with eccentricity $e=0.007$, inclination $i=0.2$ degrees, and semimajor axes distributed in two rings between $0.977 \mathrm{AU}$ and $1.023 \mathrm{AU}$. We evolve the system through a single close encounter between the planet and each test particle, to determine the fraction of test particles accreted by the planet.

When the planet has a physical radius of $10^{5} \mathrm{~km}$, Greenzweig \& Lissauer (1990) report an accretion fraction $f_{\text {acc }}=0.140$. In their validation of the SyMBA code, Duncan, Levison \& Lee (1998) estimate $f_{\text {acc }}=0.10 \pm 0.02$. For a planetary radius of $5,200 \mathrm{~km}$, the derived accretion fractions are $f_{\text {acc }}=0.009$ (Greenzweig \& Lissauer 1990) and $f_{\text {acc }}=0.008 \pm 0.002$ (Duncan, Levison $\&$ Lee 1998). Using the merger criterion specified in $\S 3$ (eq. [4]), our results are $f_{\text {acc }}=0.138 \pm 0.002$ (Poisson errors with 50,000 test particles) for the $10^{5} \mathrm{~km}$ radius planet and $f_{\text {acc }}=0.008 \pm 0.001$ for the $5,200 \mathrm{~km}$ radius planet.

A more extreme test of the merger algorithm is to simulate the high-speed collision between two counterrotating objects. We consider a small (cm-size) projectile and a larger target of some specified radius, $r$, both with a density of $1 \mathrm{~g} / \mathrm{cm}^{3}$. The two bodies initially are at $1 \mathrm{AU}$ on opposite sides of the Sun and are on colliding circular orbits. The code calculates trajectories with a fixed number of timesteps over a time interval which is randomly distributed at values just greater than $0.25 \mathrm{yr}$. We run repeated trials and find the minimum number of low-resolution timesteps, $n_{t 95}$, required to achieve a $95 \%$ success rate in detecting collisions.

Figure 6 illustrates our results. For targets with $r<1,000 \mathrm{~km}, n_{t 95}$ is determined by the errors in the interpolation of position between the endpoints of each timestep, and scales approximately as $n_{t 95} \sim r^{-1 / 3}$. In our planet formation simulations reported here, we typically take $O(100)$ timesteps per orbit and therefore can expect to resolve high-speed collisions between $1,000 \mathrm{~km}$ objects. If gravitational interactions between target and projectile become important, as in cases with large bodies and slow impact speeds, then the adaptive integrator takes high-resolution timesteps, increasing the accuracy of the merger algorithm. This effect is illustrated in Figure 6, which shows a dramatic decrease in $n_{t 95}$ for targets of size $100 \mathrm{~km}$ or larger, depending on the adaptive code's error tolerance. In practice, our $N$-bodies typically have radii greater than 1,000 km and have impact speeds which are more than an order of magnitude smaller than in a counterrotating collision. Thus, our code should accurately resolve mergers in planet formation simulations. Next, we test this assertion explicitly. 


\section{Terrestrial Planet Formation}

To illustrate the behavior of the hybrid code for less idealized conditions, we consider calculations of terrestrial planet formation at $1 \mathrm{AU}$. We compare our results with two sets of published calculations. Spaute et al. (1991) and Weidenschilling et al. (1997) use a multi-annulus code which evolves the masses and orbital properties of planetesimals with a coagulation code and follows the evolution of discrete planetary embryos with a Monte Carlo algorithm. As in our calculations, their code allows interactions between the planetesimals and the embryos. They evolve planetesimals for $1 \mathrm{Myr}$ in two configurations, 0.86-1.14 AU and 0.5-1.5 AU. Here, we use a grid at 0.84-1.16 AU and compare the Weidenschilling et al. (1997) results with our calculations from the pure coagulation code and from the hybrid code.

Chambers (2001) uses a three-dimensional $N$-body code to consider the final phase of terrestrial planet formation at 0.4-2 AU. These calculations begin with 150-160 lunar-mass to Mars-mass embryos and follow the collisional evolution for 200-500 Myr. Here, we adopt a grid at 0.4-2 AU and compare the outcomes of two pure $N$-body calculations and many hybrid calculations with the Chambers (2001) results.

We begin with results for $a=0.84-1.16$ AU. In these calculations, the grid has 32 radial zones each containing an initial distribution of planetesimals with radii of 1-4 km. The planetesimals have initial surface density $\Sigma=16(a / 1 \mathrm{AU})^{-3 / 2} \mathrm{~g} \mathrm{~cm}^{-2}$, eccentricity $e_{0}=10^{-4}$, and inclination $i_{0}$ $=6 \times 10^{-5}$. The calculations include gas drag but do not allow fragmentation.

The initial stages of pure coagulation and hybrid calculations are identical. In a few thousand

years, objects grow to radii of $10-20 \mathrm{~km}$. After $\sim 10^{4} \mathrm{yr}$, objects reach sizes of $100-300 \mathrm{~km}$. Because the timescales for dynamical friction and viscous stirring are shorter than the growth timescales, the largest objects have nearly circular orbits while the smallest objects have eccentric orbits. Runaway growth then yields a handful of $1000-3000 \mathrm{~km}$ objects. As runaway growth proceeds, viscous stirring continues to heat up the orbits of the smallest objects faster than the smallest objects damp the velocities of the largest objects. Thus, gravitational focusing factors decrease, accretion slows, and runaway growth ends. The evolution then enters a period of 'oligarchic growth,' where all of the largest objects grow at roughly the same rate (Kokubo \& Ida 1996,1998,2002).

During oligarchic growth, the growth of the largest objects in the hybrid calculations differs from the path followed in the pure coagulation models. In the hybrid models, oligarchs grow slowly until their orbits begin to cross (Kokubo \& Ida 2002; Kominami \& Ida 2002; Kenyon \& Bromley 2006). Once orbits cross, chaotic growth leads to a rapid merger rate and the formation of several 'super-oligarchs' that accrete most of the leftover planetesimals. The super-oligarchs accrete some of the remaining lower mass oligarchs and scatter the rest out of the grid (Kenyon \& Bromley 2006).

In pure coagulation models, the orbits of oligarchs are not allowed to cross. These oligarchs slowly accrete all of the planetesimals within their gravitational reach. Oligarchs rarely accrete 
other oligarchs. After 10-100 Myr, pure coagulation models have more lower mass oligarchs than the hybrid models. However, the largest oligarchs are less massive than their counterparts in the hybrid calculations.

Figures 7 and 8 compare some results from two calculations at specific times. Figure 8 of Weidenschilling et al. (1997) shows results for a similar calculation. In every case, the mass of the largest object is $\sim 5-6 \times 10^{24} \mathrm{~g}$ at $10^{4} \mathrm{yr}, \sim 3-4 \times 10^{26} \mathrm{~g}$ at $10^{5} \mathrm{yr}$, and $\sim 10^{27} \mathrm{~g}$ at $10^{6} \mathrm{yr}$. At early times, $e$ and $i$ decline monotonically with increasing mass. At later times, $e$ and $i$ have bimodal distributions: objects with $m \lesssim 10^{24}$ g have large $e$ and $i$, while more massive objects have much smaller $e$ and $i$. The orbital anisotropy, $i / e$, follows a similar evolution, with $i / e \sim 0.4$ at early times and $i / e \sim 0.1-0.15$ at late times. Near the end of the calculations, the largest objects lie in a flattened disk, with $i / e \approx 0.02-0.03$ (see also Weidenschilling et al. 1997).

Figure 9 illustrates the evolution of the eccentricity in more detail. At $10^{4} \mathrm{yr}$, dynamical friction maintains low $e$ for the largest bodies. Because planets form fastest at the inner edge of the grid, small objects at the inner edge have larger $e$ than small objects at the outer outer edge. At $10^{5} \mathrm{yr}$, dynamical friction still maintains low $e$ for the largest bodies. Because most of the small objects have grown to $100 \mathrm{~km}$ radius, these objects have slightly smaller $e$ than other objects with $m<10^{24} \mathrm{~g}$. At $10^{6} \mathrm{yr}$, objects with $m>10^{26} \mathrm{~g}$ have low $e \sim 0.02$, while lower mass objects have higher $e \sim 0.1$. These results compare favorably with Figure 9 of Weidenschilling et al. (1997).

We begin our version of the Chambers (2001) calculations in a grid of 40 annuli at $a=0.4-2$ AU. For the hybrid calculations, the annuli contain an initial distribution of planetesimals with radii of $4-15 \mathrm{~km}$. The pure $N$-body model starts with 160 'moons' with a mass of $10^{26} \mathrm{~g}$. In both cases, the objects have $e_{0}=10^{-4}$ and $i_{0}=6 \times 10^{-5}$. To provide some contrast with previous calculations, we adopt an initial surface density of planetesimals or moons, $\Sigma=x \Sigma_{0}(a / 1 \mathrm{AU})^{-1}$ with $\Sigma_{0}=8 \mathrm{~g}$ $\mathrm{cm}^{-2}$ and $x=0.25-2$, instead of the usual $\Sigma \propto a^{-3 / 2}$. Although the final configuration of planets depends on the initial surface density gradient, the general evolution is fairly independent of $\Sigma$.

The large radial extent of these calculations allows us to illustrate the sensitivity of planet formation to the heliocentric distance. Because the timescale for growth by coagulation is $t \propto$ $\Sigma / P \propto a^{5 / 2}$, planets grow fastest at the inner edge of the grid (Lissauer 1987). In hybrid models with $x=1$, objects with radii of $\sim 200 \mathrm{~km}$ form in $\sim 10^{3}$ yr at $0.4 \mathrm{AU}$, in $\sim 10^{4}$ yr at $0.95 \mathrm{AU}$, and in $\sim 10^{5} \mathrm{yr}$ at $2 \mathrm{AU}$. Oligarchs with masses of $\sim 10^{26} \mathrm{~g}$ form on a timescale

$$
t_{o} \approx 1.5 \times 10^{5} x^{-1}(a / 1 \mathrm{AU})^{5 / 2}
$$

Once large objects start to form, the transition from runaway to oligarchic to chaotic growth proceeds in several waves propagating from the inner disk to the outer disk (Kenyon \& Bromley 2006). As oligarchs start to form at the outer edge of the grid, dynamical interactions between oligarchs begin at the inner edge. Several orbit crossings lead to dynamical interactions between all oligarchs and a rapid increase in the merger rate. A few large oligarchs gradually accrete many of the smaller oligarchs, leading to a configuration with several Earth-mass planets and a few 
Mars-mass 'leftovers.'

In the moons calculations, dynamical interactions between the 160 original oligarchs dominate the entire evolutionary sequence. In the first $\sim 10^{4} \mathrm{yr}$, five mergers start to produce large oligarchs in the inner disk. The number of oligarchs declines to 146 in $10^{5} \mathrm{yr}$, to 115 in $1 \mathrm{Myr}$, and to 55 in $10 \mathrm{Myr}$. By $10 \mathrm{Myr}$, the largest objects in the inner disk reach masses of $0.2-0.3 M_{\oplus}$ and slowly accrete the remaining moons. In the outer disk, the smaller surface density and viscous stirring lead to a smaller merger rate. After $\sim 100 \mathrm{Myr}$, only $\sim 20$ oligarchs remain in the outer disk.

To compare our results with Chambers (2001), we consider measures of the orbital elements of final 'solar systems' from several simulations. In our direct $N$-body and hybrid calculations, the time variation of the mass-weighted eccentricity $\bar{e}$ (Fig. 10) follows a standard pattern (see Fig. 5 of (Chambers 2001)). During runaway and oligarchic growth, protoplanets stir their surroundings and $\bar{e}$ increases. At 10-100 Myr, the orbits of oligarchs cross, which further excites orbital eccentricity and produces sharp peaks in $\bar{e}$. Mergers between oligarchs reduce $\bar{e}$. Once a few remaining planets have fairly stable orbits, $\bar{e}$ settles to $0.05-0.15$.

Hybrid models yield larger variations in $\bar{e}$ than $N$-body calculations of large objects. Because objects grow to the promotion mass throughout a hybrid calculation, the merger phase lasts longer and produces more frequent resonant interactions at later times compared to pure $N$-body models. Thus, we often observe several peaks in the evolution of $\bar{e}$ for hybrid models compared to a single peak in pure $N$-body calculations.

Figure 11 shows the relation between the eccentricity and mass of the planets produced in our calculations. Our models yield a range in mass from $\sim 0.01 M_{\oplus}$ to $\sim 2-3 M_{\oplus}$. For $\sim 30$ planets with masses, $m \gtrsim 0.1 M_{\oplus}$, the orbital eccentricity is not correlated with the mass. Chambers (2001) derived a similar result for $\sim 50$ planets. However, our calculations yield a reasonably large ensemble of lower mass planets with $m \sim 0.01-0.1 M_{\oplus}$. In the full ensemble of planets with $m \sim$ 0.01-3 $M_{\oplus}$, there is a small, but significant trend of decreasing eccentricity with increasing planet mass. From the Spearman rank and Kendall's $\tau$ tests (Press et al. 1992), the probability that the derived distribution of $m(e)$ is random is $\sim 2 \times 10^{-3}$.

To compare this result to our Solar System, we restrict the mass range to $0.05-2 M_{\oplus}$. For our ensemble of $\sim 40$ planets, the Spearman rank and Kendall's $\tau$ probabilities are $\sim 0.1$. These tests yield probabilities of $\sim 0.15-0.2$ for the 4 terrestrial planets in our Solar System. We conclude that the weak correlation of $e$ with $m$ is consistent with architecture of our Solar System.

As a final comparison with Chambers (2001), we calculate several statistical parameters to characterize the final configurations of our models. For a system with $N$ oligarchs,

$$
S_{m}=m_{l} / m_{t}
$$

measures the fraction of the mass in the largest object, where $m_{l}$ is the mass of the largest oligarch 
and $m_{t}$ is the total mass in the grid. The orbital spacing statistic is

$$
S_{s}=\frac{6}{N-1}\left(\frac{a_{\max }-a_{\min }}{a_{\max }+a_{\min }}\right)\left(\frac{3 m_{c}}{2 \bar{m}}\right)^{1 / 4}
$$

where $\bar{m}$ is the average mass of an oligarch, $m_{c}$ is the mass of the central star, $a_{\text {min }}$ is the minimum semimajor axis of an oligarch, and $a_{\max }$ is the maximum semimajor axis of an oligarch. To measure the degree of orbital excitation of a planet, Laskar (1997) describes the angular momentum deficit, the difference between the z-component of the angular momentum of an orbit and a circular orbit with the same semimajor axis. Chambers (2001) generalizes this definition as the sum over all planets,

$$
S_{d}=\frac{\sum_{j=1}^{N} m_{j} \sqrt{a_{j}}\left[1-\sqrt{\left(1-e_{j}^{2}\right)} \operatorname{cosi}_{j}\right]}{\sum_{j=1}^{N} m_{j} \sqrt{a_{j}}} .
$$

Finally, the mass concentration statistic

$$
S_{c}=\max \left(\frac{\sum_{j=1}^{N} m_{j}}{\sum_{j=1}^{N} m_{j}\left[\log \left(a / a_{j}\right)^{2}\right]}\right)
$$

measures whether mass is concentrated in a few massive objects (large $S_{c}$; as in the Earth and Venus) or many low mass objects (small $S_{c}$; as in the Kuiper belt and scattered disk of the outer solar system).

Table 1 compares results for our 14 hybrid calculations and our two 'moons' calculations with statistics for the inner Solar System. For calculations with $x=1$, our moons calculations yield roughly the same outcome as Chambers (2001). The mass of the largest object and the statistics agree well with those in Table 1 of Chambers (2001). Because we began with a shallower surface density gradient, our planetary systems have more massive objects and more oligarchs at larger heliocentric distances than Chambers (2001).

Our hybrid models for $x=1$ yield results similar to the moons calculations. Our most massive object has a mass of 1.1-1.8 $M_{\oplus}$ and contains $40 \%$ to $80 \%$ of the initial mass in the grid. The calculations typically produce 1-2 other massive objects comparable in mass to Venus and several less massive objects with masses more similar to Mars. For models with longer evolution times, the number of Mars-mass objects declines considerably from 100 Myr to 500 Myr. The statistical parameters agree well with the Chambers (2001) results. The planets in all of our model solar systems have more eccentric orbits than the planets in the Solar System. However, the other statistics agree rather well with those in the Solar System, including one model that is more concentrated than the Solar System.

The outcomes of our calculations are sensitive to the initial mass in the grid. Models with $x$ $=0.25$ produce very low mass planets, $\sim 0.1 M_{\oplus}$, compared to models with $x=1-2, \sim 1-2 M_{\oplus}$. The lower mass models also yield more planets on more circular orbits and have a smaller fraction of the total mass in the largest object. 


\section{CONCLUSION}

In this paper we describe a hybrid $N$-body coagulation code for planet formation. We put the $N$-body part through a battery of tests to assess its performance in planetary dynamics simulations. Validation tests of the coagulation algorithm appear elsewhere (e.g., Kenyon \& Bromley 2001). We provide tests of the hybrid method by comparing it to coagulation simulations and $N$-body output separately. We demonstrate that all three methods quantitatively reproduce published results for the formation of rocky planets by mergers of $O\left(10^{12}\right) \mathrm{km}$-sized (Weidenschilling et al. 1997) or $O(100)$ moon-sized (Chambers 2001) planetesimals. Table 2 gives a summary of these tests, with references to other published work.

Although our intent here is simply to describe our method, the hybrid code now has the potential to match observations of the Solar System and debris disks (e.g. Kenyon \& Bromley 2004a,b). In addition to results on terrestrial planets in $\S 5$, Kenyon \& Bromley (2006) summarizes results on the transition from oligarchic to chaotic growth in the terrestrial zone. Kenyon \& Bromley (2004c) shows how a stellar encounter can produce Sedna-like orbits in the outer Solar System and outlines how observations might distinguish between locally-produced and captured Sednas. Future papers will describe more complete results on planet formation in the terrestrial zone and the trans-Neptunian region.

We acknowledge a generous allotment, $\sim 20$ cpu years, of computer time on the Silicon Graphics Origin-2000 'Alhena' through funding from the NASA Offices of Mission to Planet Earth, Aero-

nautics, and Space Science. Advice and comments from M. Geller are greatly appreciated! The NASA Astrophysics Theory Program supported part of this project through grant NAG5-13278. 


\section{REFERENCES}

Aarseth, S. J. 1985, IAU Symp. 113: Dynamics of Star Clusters, 113, 251

Backman, D. E., \& Paresce, F. 1993, in Protostars and Planets III, eds. E. H. Levy \& J. I. Lunine, Tucson, Univ of Arizona, p. 1253

Barnes, J. \& Hut, P. 1986, Nature, 324, 446

Barton, E. J., Bromley, B. C., \& Geller, M. J. 1999, ApJ, 511, L25

Beckwith, S. V. W. 1999, in The Physics of Star Formation and Early Stellar Evolution, ed. by C. J. Lada \& N. Kylafis (Dordrecht, Kluwer), p. 579

Chambers, J. E. 2001, Icarus, 152, 205

Duncan, M. J. \& Lissauer, J. J. 1998, Icarus, 134, 303

Duncan, M. J., Levison, H. F., \& Lee, M. H. 1998, AJ, 116, 2067

Encke, M. 1852, MNRAS, 13, 2

Fukushima, T. 1996, AJ, 112, 1263

Geller, M. J. \& Huchra, J. P. 1983, ApJS, 52, 61

Greaves, J. S. et al. 1998, ApJ, 506, L133

Hayashi, C. 1981, Prog Theor Phys Suppl, 70, 35

Hornung, P., Pellat, R., \& Barge, P. 1985, Icarus, 64, 295

Huchra, J. P. \& Geller, M. J. 1982, ApJ, 257, 423

Hut, P. \& Makino, J. 1999, Science, 283, 501

Hut, P., Makino, J., \& McMillan, S. 1995, ApJ, 443, L93

Ida, S., \& Makino, J. 1992, Icarus, 96, 107

Ida, S., \& Makino, J. 1993, Icarus, 106, 210

Jayawardhana, R. et al. 1998, ApJ, 503, L79

Jewell, G. M. \& Alexander, S. G. 1996, BAAS, 28, 1107

Kalas, P., Larwood, J., Smith, B. A., \& Schultz, A. 2000, ApJ, 530, L133

Kenyon, S. J., \& Bromley, B. C. 2001, AJ, 121, 538 
Kenyon, S. J. \& Bromley, B. C. 2002, ApJ, 577, L35

Kenyon, S. J., \& Bromley, B. C. 2002, AJ, 123, 1757

Kenyon, S. J., \& Bromley, B. C. 2004, AJ, 127, 513

Kenyon, S. J., \& Bromley, B. C. 2004b, ApJ, 602, L133

Kenyon, S. J., \& Bromley, B. C. 2004c, Nature, 432, 598

Kenyon, S. J., \& Bromley, B. C. 2006, AJ, 131, No. 3

Kenyon, S. J., \& Luu, J. X. 1998, AJ, 115, 2136

Kenyon, S. J., \& Luu, J. X. 1999, AJ, 118, 1101

Kinoshita, H., Yoshida, H., \& Nakai, H. 1991, Celestial Mechanics and Dynamical Astronomy, 50, 59

Koerner, D. W., Ressler, M. E., Werner, M. W., \& Backman, D. E. 1998, ApJ, 503, L83

Koerner, D. W., Sargent, A. I., \& Ostroff, N. A. 2001, ApJ, 560, L181

Kokubo, E. \& Ida, S. 1995, Icarus, 114, 247

Kokubo, E., \& Ida, S. 1996, Icarus, 123, 180

Kokubo, E., \& Ida, S. 1998, Icarus, 131, 171

Kokubo, E., \& Ida, S. 2000, Icarus, 143, 15

Kokubo, E., \& Ida, S. 2002, ApJ, 581, 666

Kokubo, E., Kominami, J., \& Ida, S. 2006, ApJ, in press

Kokubo, E., Yoshinaga, K., \& Makino, J. 1998, MNRAS, 297, 1067

Kominami, J. \&; Ida, S. 2002,Icarus, 157, 43

Lada, C. J. 1999, in The Physics of Star Formation and Early Stellar Evolution, edited by C. J. Lada and N. Kylafis, Dordrecht, Kluwer, p. 143

Laskar, J. 1997, ApJ, 317, L75

Lissauer, J. J. 1987, Icarus, 69, 249

Marcy, G. W. \& Butler, R. P. 2000, PASP, 112, 137

McMillan, S. L. W. \& Aarseth, S. J. 1993, ApJ, 414, 200 
Ohtsuki, K., Stewart, G. R., \& Ida, S. 2002, Icarus, 155, 436

Press, W. H., Flannery, B. P., Teukolsky, S. A., \& Vetterling, W. T. 1992, Numerical Recipes, The Art of Scientific Computing, Cambridge, Cambridge

Quinlan, G. D. \& Tremaine, S. D. 1990, BAAS, 22, 949

Safronov, V. S. 1969, Evolution of the Protoplanetary Cloud and Formation of the Earth and Planets, Nauka, Moscow [Translation 1972, NASA TT F-677]

Saha, P. \& Tremaine, S. 1992, AJ, 104, 1633

Shefer, V. A. 2002, Celestial Mechanics and Dynamical Astronomy, 82, 19

Skeel, R. D., \& Biesiadecki, J. J. 1994, Ann. Numer. Math., 1, 191

Spaute, D., Weidenschilling, S. J., Davis, D. R., \& Marzari, F. 1991, Icarus, 92, 147

Stewart, G. R., \& Ida, S. 2000, Icarus, 143, 28

Vasilev, A. M. 1982, Komety i Meteory, 33, 39

Wahhaj, Z., Koerner, D. W., Ressler, M. E., Werner, M. W., Backman, D. E., \& Sargent, A. I. 2003, ApJ, 584, L27

Weidenschilling, S. J. 1980, Icarus, 44, 172

Weidenschilling, S. J. 1989, Icarus, 80, 179

Weidenschilling, S. J., Spaute, D., Davis, D. R., Marzari, F., \& Ohtsuki, K. 1997, Icarus, 128, 429

Wetherill, G. W., \& Stewart, G. R. 1993, Icarus, 106, 190

Wisdom, J. \& Holman, M. 1991, AJ, 102, 1528

Yoshida, H. 1990, Phys. Lett. A, 150, 262 
Table 1. Statistics for Planetary Systems at 0.4-2 AU

\begin{tabular}{lccccccc}
\hline \hline$\Sigma / \Sigma_{0}$ & $t_{\text {evol }}(\mathrm{Myr})$ & $\mathrm{N}$ & $M_{l}\left(M_{\oplus}\right)$ & $S_{m}$ & $S_{s}$ & $S_{d}$ & $S_{c}$ \\
\hline 0.25 & 100 & 9 & 0.1 & 0.198 & 19.9 & 0.0031 & 26.4 \\
0.25 & 300 & 10 & 0.1 & 0.213 & 22.7 & 0.0029 & 24.7 \\
0.50 & 100 & 10 & 0.4 & 0.292 & 20.1 & 0.0058 & 26.1 \\
0.50 & 100 & 8 & 0.4 & 0.314 & 25.1 & 0.0094 & 21.9 \\
1.00 & 100 & 6 & 1.3 & 0.474 & 27.7 & 0.0090 & 33.4 \\
1.00 & 100 & 8 & 1.0 & 0.526 & 23.4 & 0.0118 & 45.8 \\
1.00 & 100 & 5 & 1.1 & 0.428 & 35.6 & 0.0131 & 33.4 \\
1.00 & 100 & 6 & 1.4 & 0.498 & 28.3 & 0.0093 & 27.0 \\
1.00 & 200 & 5 & 1.1 & 0.371 & 28.7 & 0.0121 & 22.4 \\
1.00 & 200 & 5 & 1.8 & 0.644 & 32.0 & 0.0499 & 52.6 \\
1.00 & 500 & 2 & 2.1 & 0.778 & 36.1 & 0.0228 & 119.4 \\
2.00 & 100 & 5 & 2.4 & 0.521 & 19.3 & 0.0328 & 38.6 \\
2.00 & 100 & 6 & 2.0 & 0.348 & 24.6 & 0.0292 & 25.7 \\
& & & & & & & \\
1.00 & 100 & 21 & 0.8 & 0.256 & 14.2 & 0.0136 & 17.1 \\
1.00 & 100 & 15 & 0.9 & 0.417 & 28.8 & 0.0119 & 25.4 \\
& & & & & & & \\
MVEM & & 4 & 1.0 & 0.509 & 37.7 & 0.0018 & 89.9 \\
\hline
\end{tabular}


Table 2. Summary of Numerical Tests

\begin{tabular}{|c|c|c|}
\hline Test name & Result (this paper) & Result (other references) \\
\hline \multicolumn{3}{|l|}{ Tests of integration accuracy: } \\
\hline Giant planet orbit integration & Figure 1 & - \\
\hline Scaled Outer Solar System & Figure 2 & Figure 5, Duncan, Levison \& Lee (1998) \\
\hline \multicolumn{3}{|l|}{ Tests of dynamic range: } \\
\hline Binary Jupiters & Figure 3 & Figure 4, Duncan, Levison \& Lee (1998) \\
\hline Earth satellite & Figure 3 & 二 \\
\hline \multicolumn{3}{|l|}{ Planetesimal dynamics: } \\
\hline Resolving chaos & Figure 4 & - \\
\hline Two planetesimals in a swarm & Figure 5 & Figure 1, Kokubo \& Ida (1995) \\
\hline & & Figure B3, Weidenschilling et al. (1997) \\
\hline \multicolumn{3}{|l|}{ Merger algorithm: } \\
\hline $\begin{array}{l}\text { Planetary accretion }\left(R=10^{5} \mathrm{~km}\right) \\
\quad\left(f_{a c c} \text { is the accretion fraction }\right)\end{array}$ & $f_{a c c}=0.138 \pm 0.002$ & $\begin{array}{l}f_{a c c}=0.140, \text { Greenzweig \& Lissauer }(1990) \\
f_{a c c}=0.10 \pm 0.2, \text { Duncan, Levison \& Lee }(1998)\end{array}$ \\
\hline Planetary accretion $(R=5200 \mathrm{~km})$ & $f_{a c c}=0.008 \pm 0.001$ & $\begin{array}{l}f_{a c c}=0.009, \text { Greenzweig \& Lissauer }(1990) \\
f_{a c c}=0.008 \pm 0.002, \text { Duncan, Levison \& Lee }(1998)\end{array}$ \\
\hline high-speed collisions & Figure 6 & - \\
\hline \multicolumn{3}{|l|}{ Hybrid code: } \\
\hline Planet formation (coagulation) & Figures $7-9$ & Figures 8 \& 9, Weidenschilling et al. (1997) \\
\hline Terrestrial planet formation & Table 1 & Table 1, Chambers (2001) \\
\hline
\end{tabular}




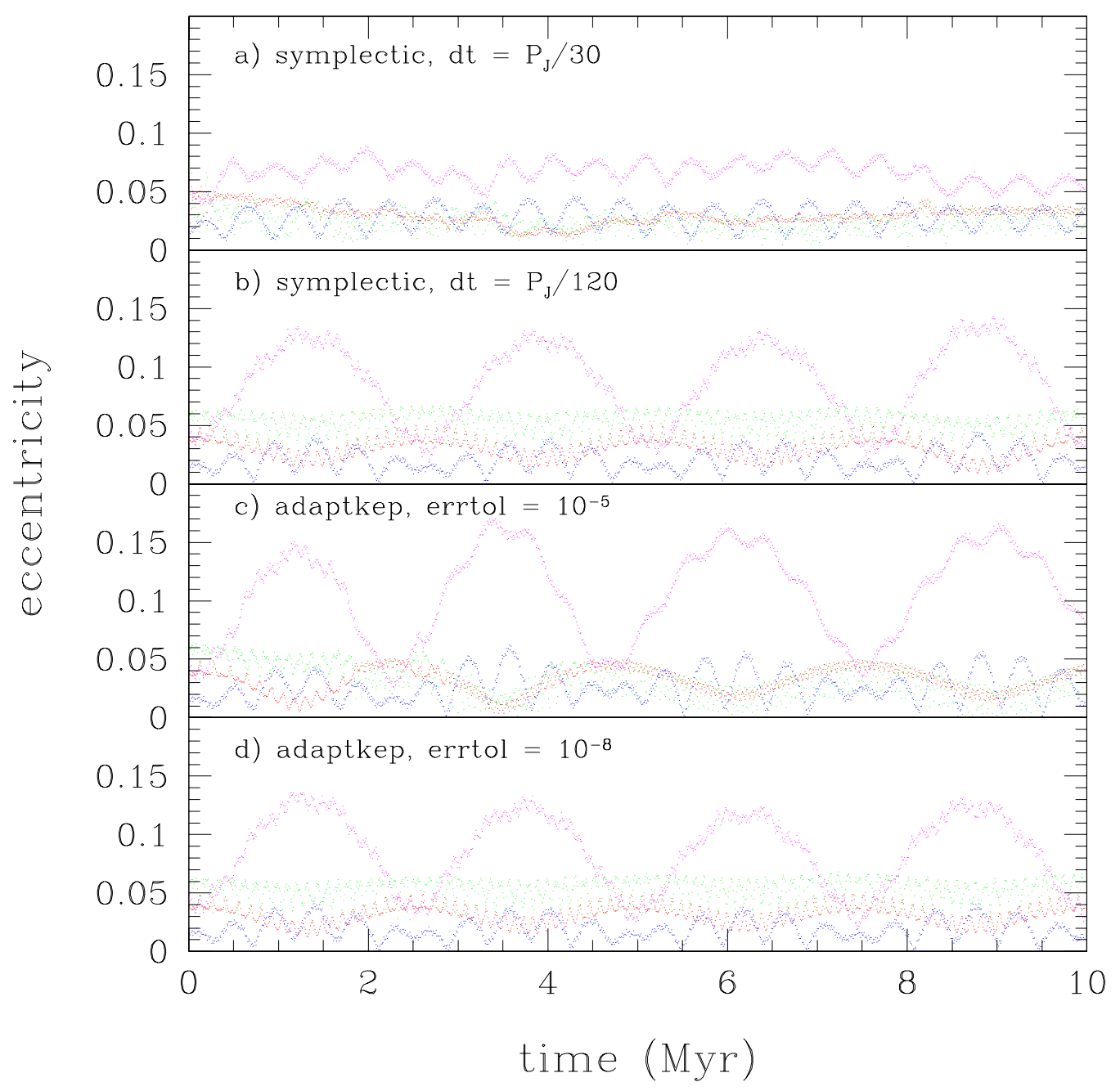

Fig. 1. - The eccentricity of the major planets derive from a symplectic code and the adaptive code. With a large timestep $d t$ of one thirtieth of the period of Jupiter's orbit $\left(P_{J}\right)$, the symplectic integrator (panel a) generates phase errors - slow drifts from the true phase-space position of the planets. These errors are reflected in the relatively limited range of eccentricities, as compared with a run with smaller timesteps (panel b). The adaptive code, with a large error tolerance, will run with large timesteps. The adaptive results (panel c) generate more realistic eccentricities compared to the small-timestep symplectic code and the small-error tolerance adaptive code (panel d). However, the adaptive code suffers from a slow drift in energy. The net fractional energy errors in the adaptive runs are $10^{-3}\left(10^{-5}\right)$ for large (small) error tolerances. 


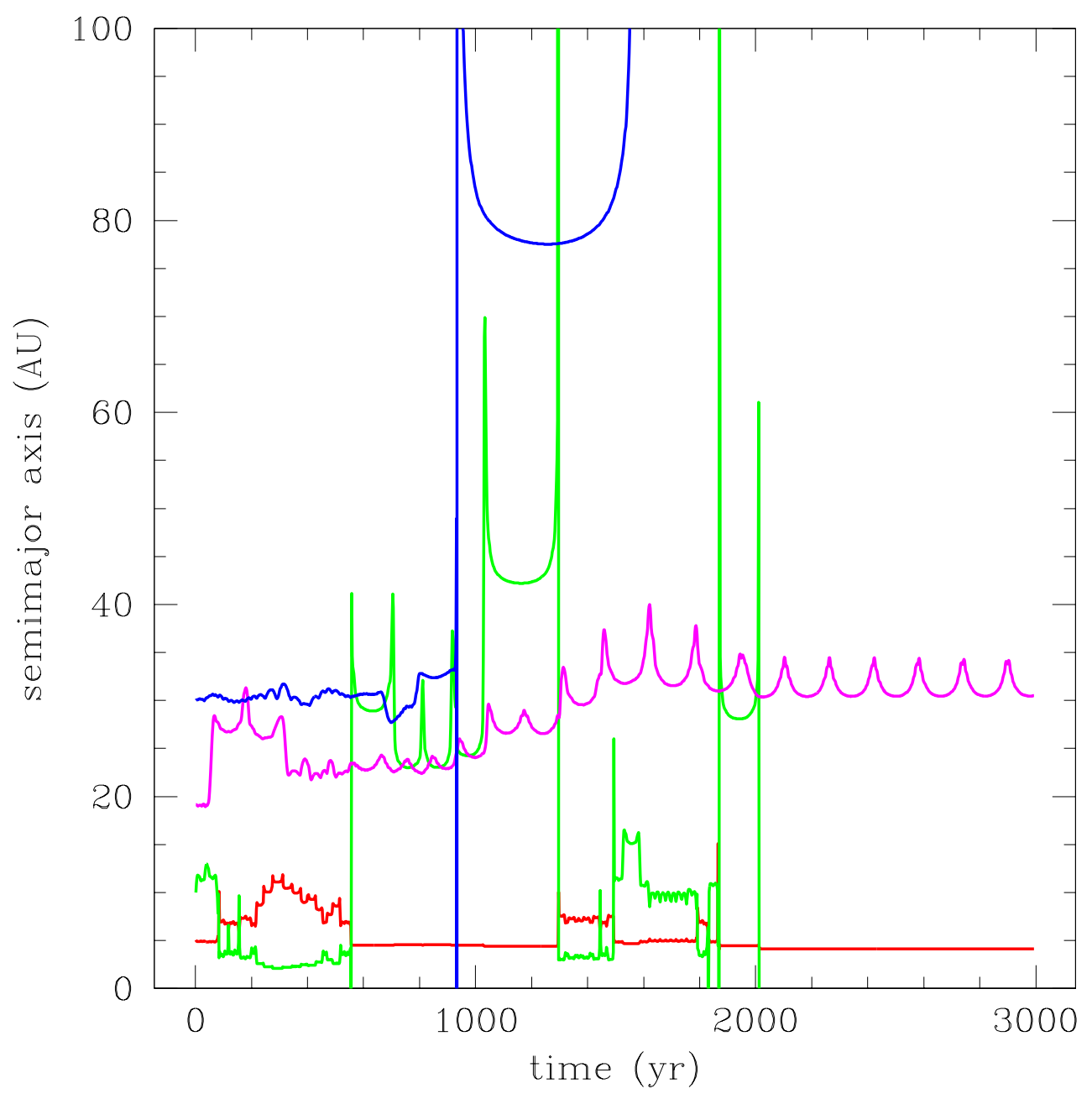

Fig. 2.- The instantaneous Keplerian semimajor axes of the major planets, with masses scaled by a factor of 50, as a function of time from a simulation with our adaptive integrator. Saturn is ejected just after 2,000 yr; Neptune has acquired an orbit with eccentricity of 0.92 and a semimajor axis of about $250 \mathrm{AU}$. The overall dynamics are highly chaotic and quite sensitive to the outcome of the initial interaction between Jupiter and Saturn in the first $100 \mathrm{yr}$ of the simulation. 


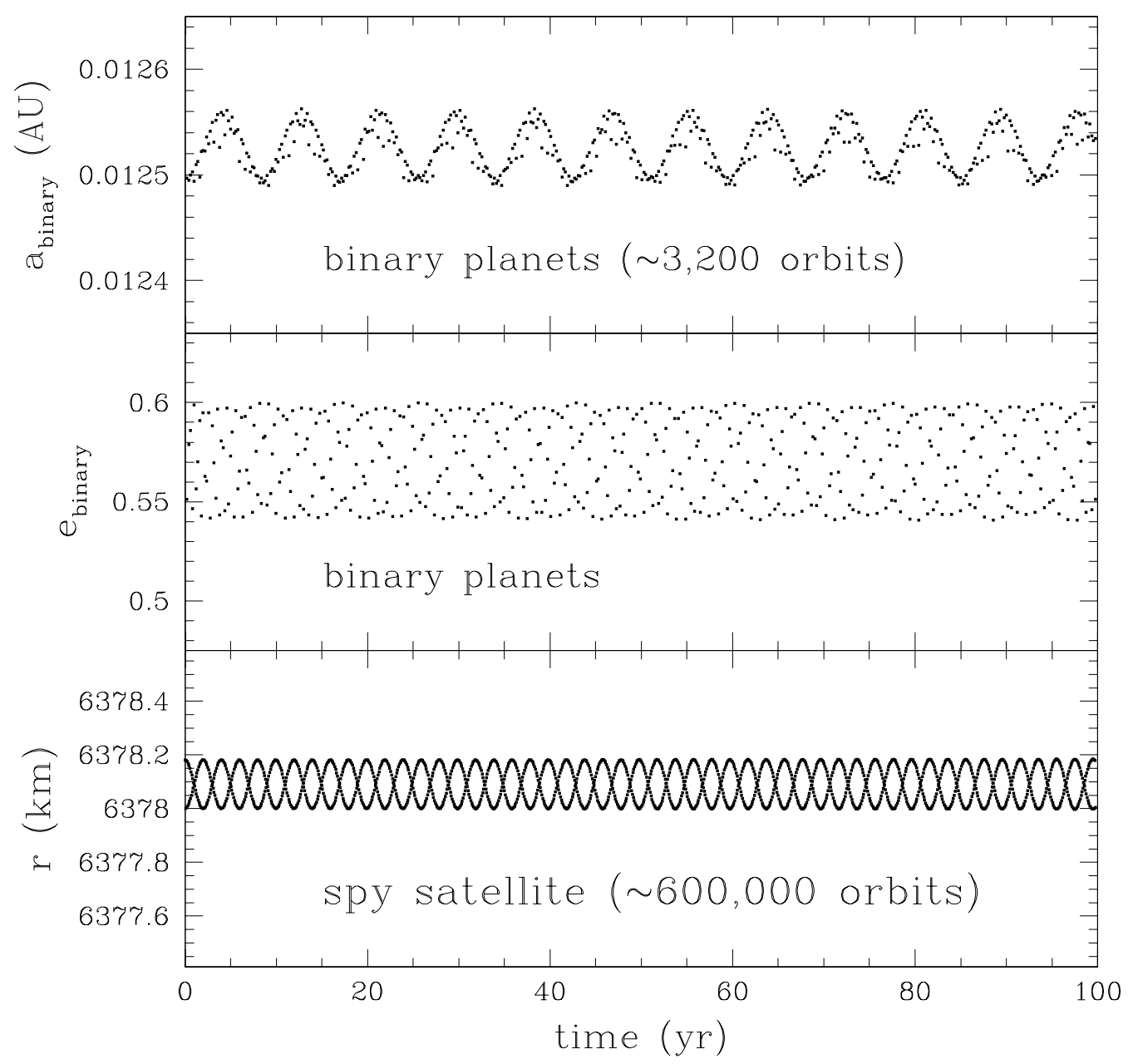

Fig. 3.- Gravitationally bound binaries orbiting a solar-mass star. The upper panel shows the semimajor axis of a pair of Jupiter-mass planets as calculated in the center-of-mass frame of the pair as they orbit the star. The binary eccentricity is $e=0.6$, as in Duncan, Levison \& Lee (1998). The lower panel is the radial position of a test-particle ("spy satellite") on a polar orbit about the center of the Earth. In this case, we assume the Earth is spherically symmetric. Each panel shows only a single quantity; the appearance of multiple curves is an artifact of sampling. 


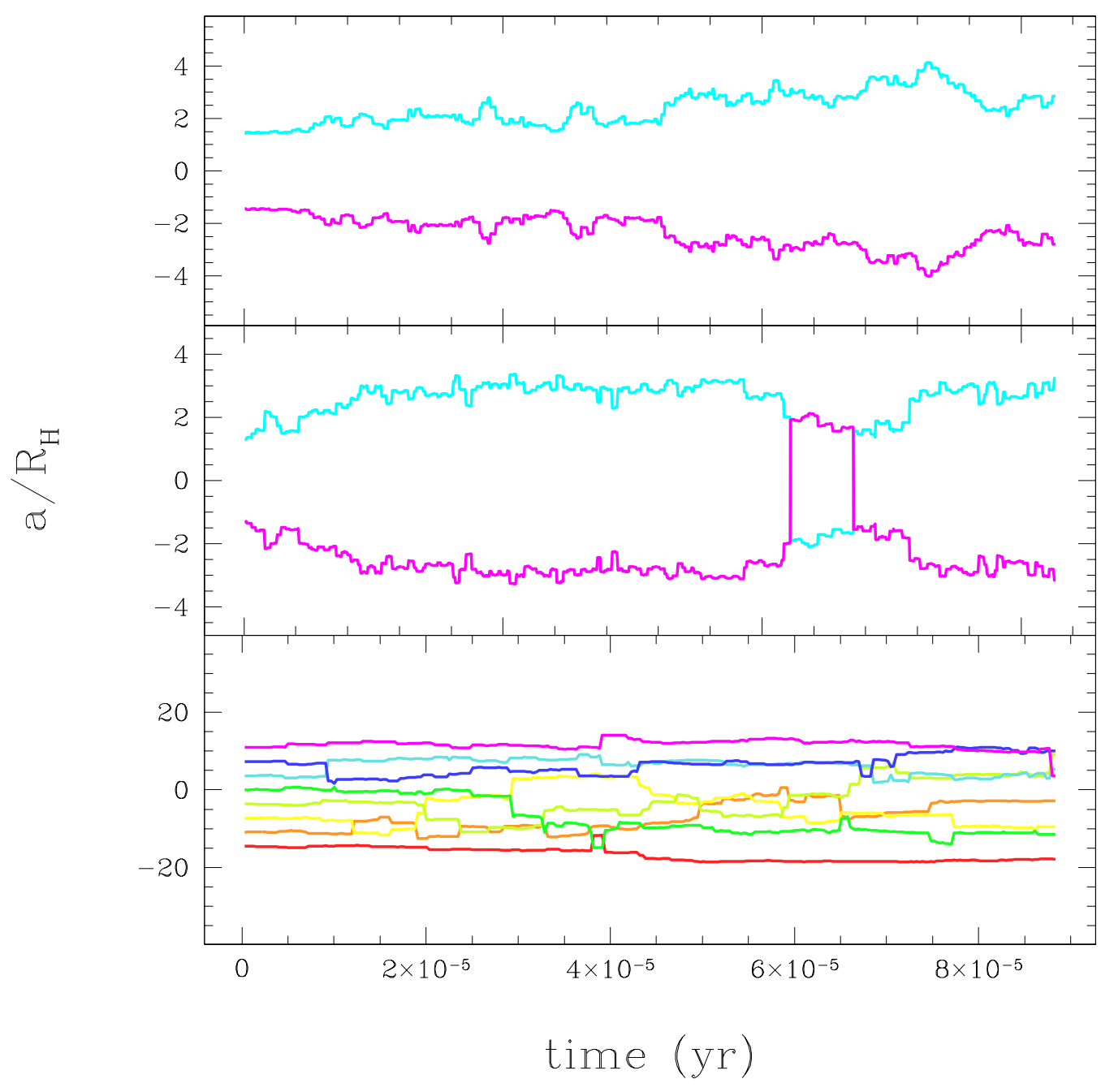

Fig. 4. - Integration of planetesimal orbits. The upper two panels show the orbital separation of a pair of $10^{26} \mathrm{~g}$ objects in units of the mutual Hill radius. In both cases the particles were placed on circular orbits near $1 \mathrm{AU}$ with separation of $2.1 \sqrt{3} r_{H}$ (top) and $1.9 \sqrt{3} r_{H}$ (middle), where $r_{H}$ is the mutual Hill radius. When the orbital separation is less than $2 \sqrt{3} r_{H}$, then the orbits will cross. The lower panel shows interactions between eight particles separated by $2.1 \sqrt{3} r_{H}$. 


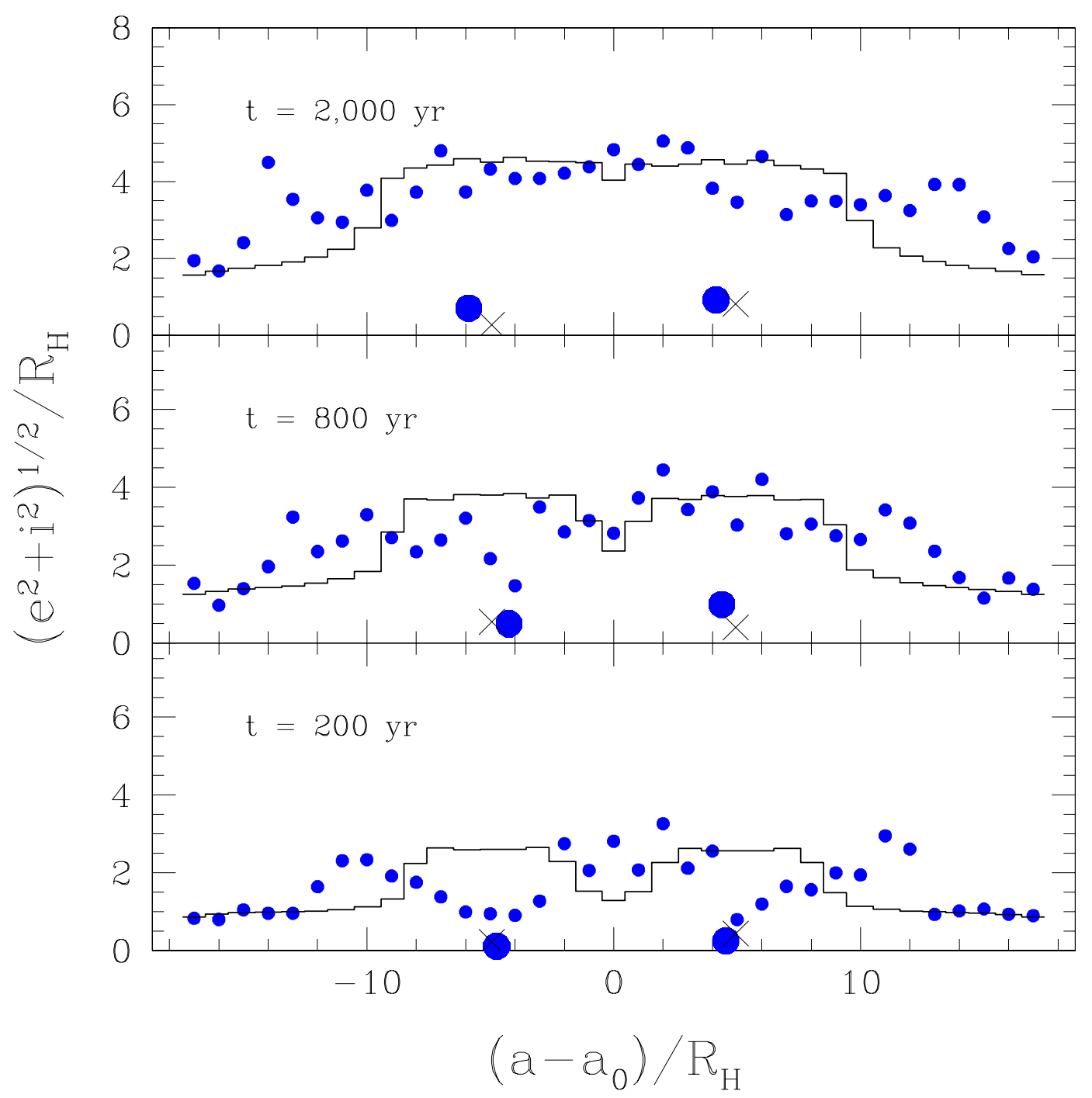

Fig. 5. - Evolution of $\left(e^{2}+i^{2}\right)^{1 / 2}$ in units of the Hill radius for two $10^{26}$ g objects and a swarm of 800 $2 \times 10^{24} \mathrm{~g}$ planetesimals. The horizontal axis gives the orbital radius relative to $a_{0}=1 \mathrm{AU}$ around a solar-type star. The large filled circles correspond to a pair of the heavy objects at $200 \mathrm{yr}$ (lower panel), $800 \mathrm{yr}$ (middle panel) and $2000 \mathrm{yr}$ (top panel). The small filled circles indicate values of $\left(e^{2}+i^{2}\right)^{1 / 2}$ for the planetesimals averaged in radial bins. The $\times$ symbols and histograms correspond to the two massive objects and planetesimals in a coagulation model (Kenyon \& Bromley 2001). 


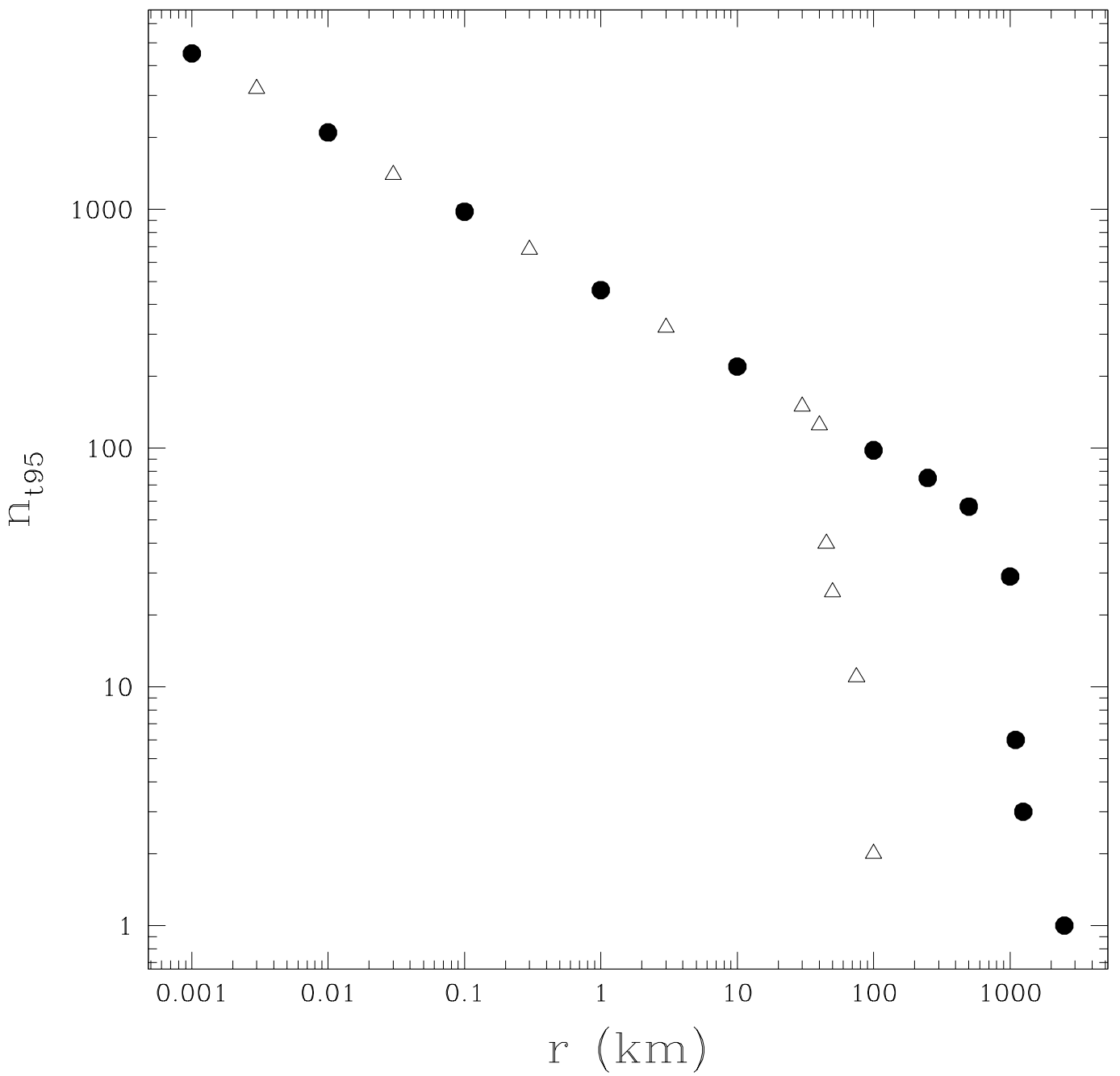

Fig. 6. - The minimum number of timesteps leading to successful pairwise collisions as a function of the planetesimal radius. Specifically, $n_{t 95}$ is the minimum number of low-resolution timesteps required to resolve a merger between a point-like projectile and a target planetesimal of radius $r$, with a success rate of $95 \%$. The target and projectile are on counterrotating, circular orbits at 1 AU, initially on opposite sides of the Sun. The error tolerance for the adaptive code is $10^{-11}$ (filled circles) and $10^{-15}$ (open triangles). The precipitous drop in $n_{t 95}$ at large $r$ in both cases results from the adaptive code's use of high-resolution timesteps as it attempts to account for the gravitational effect of the target on the projectile. 
size distribution

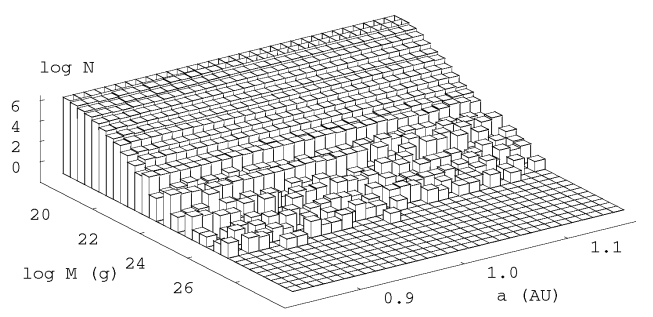

$10^{4} \mathrm{yr}$

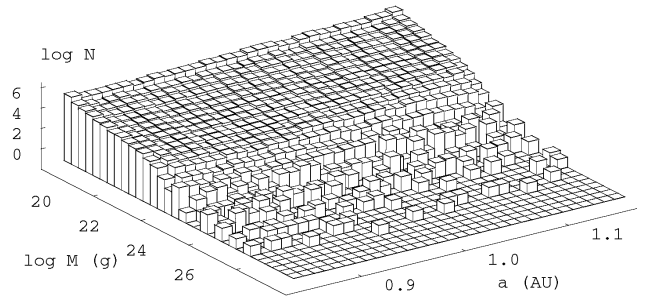

$10^{5} \mathrm{yr}$
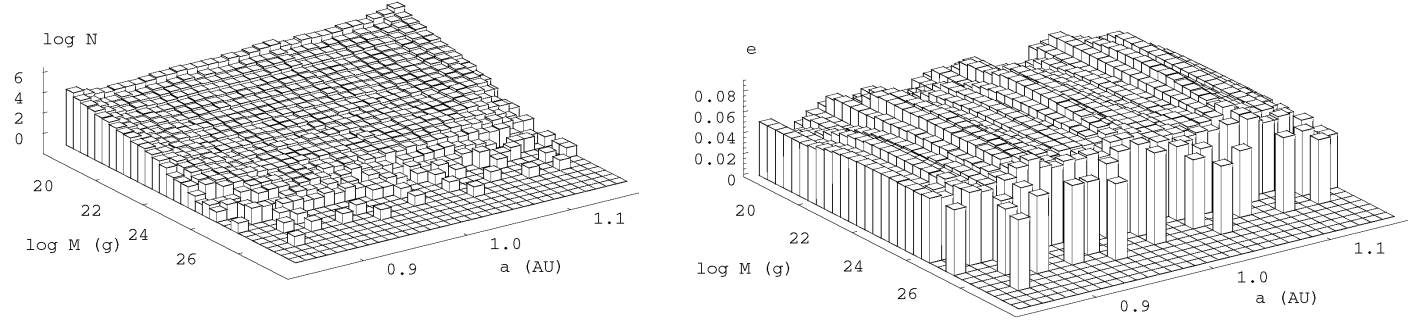

$10^{6} \mathrm{yr}$

Fig. 7.- Evolution of particle number (left panels) and eccentricity (right panels) for planetesimals orbiting a $1 \mathrm{M}_{\odot}$ star at $1 \mathrm{AU}$. The 32 annuli in the grid contain planetesimals with initial radii of $1-4 \mathrm{~km}$, initial eccentricity $e_{0}=1 \times 10^{-4}$, and a total mass of $0.6 M_{\oplus}$. The panels show the evolution of $N(m)$ and $e$ for calculations with the coagulation code at $t=10^{4}$ yr (upper panels), $t=10^{5} \mathrm{yr}$ (middle panels), and $t=10^{6} \mathrm{yr}$ (lower panels). Style adapted from Weidenschilling et al. (1997). 
size distribution

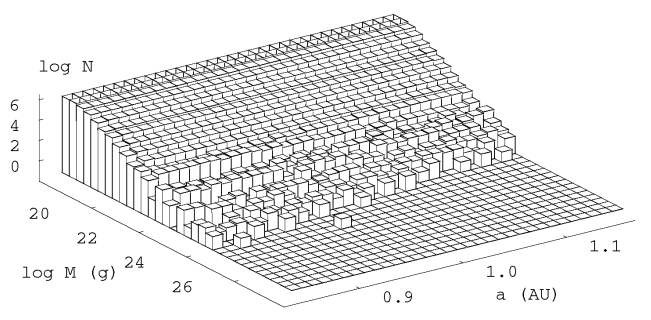

$10^{4} \mathrm{yr}$
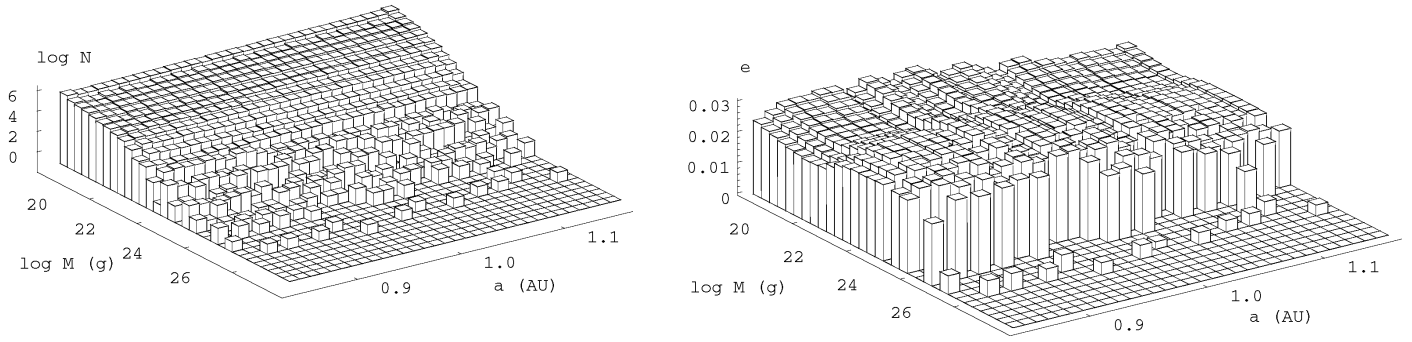

$10^{5} \mathrm{yr}$
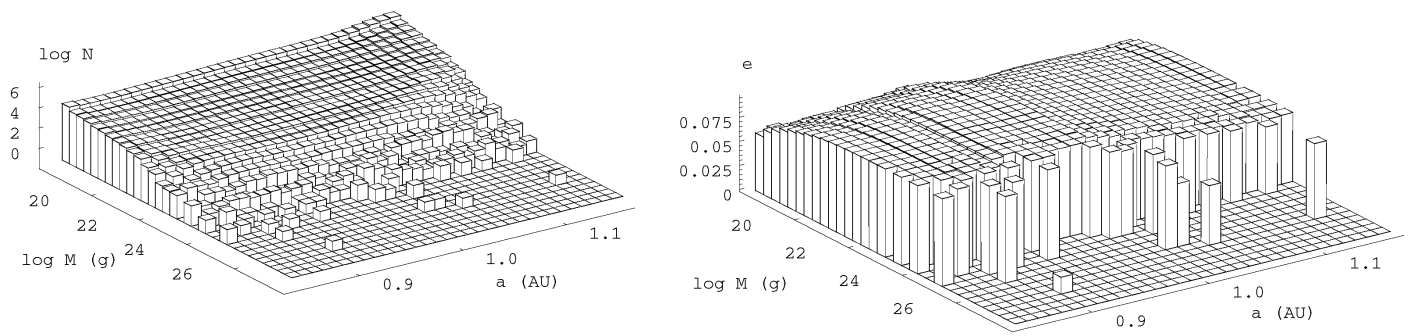

$10^{6} \mathrm{yr}$

Fig. 8.- Evolution of particle number (left panels) and eccentricity (right panels) for planetesimals in orbit around a $1 \mathrm{M}_{\odot}$ star at $1 \mathrm{AU}$, as in Figure 7 , but for calculations with the hybrid code. 


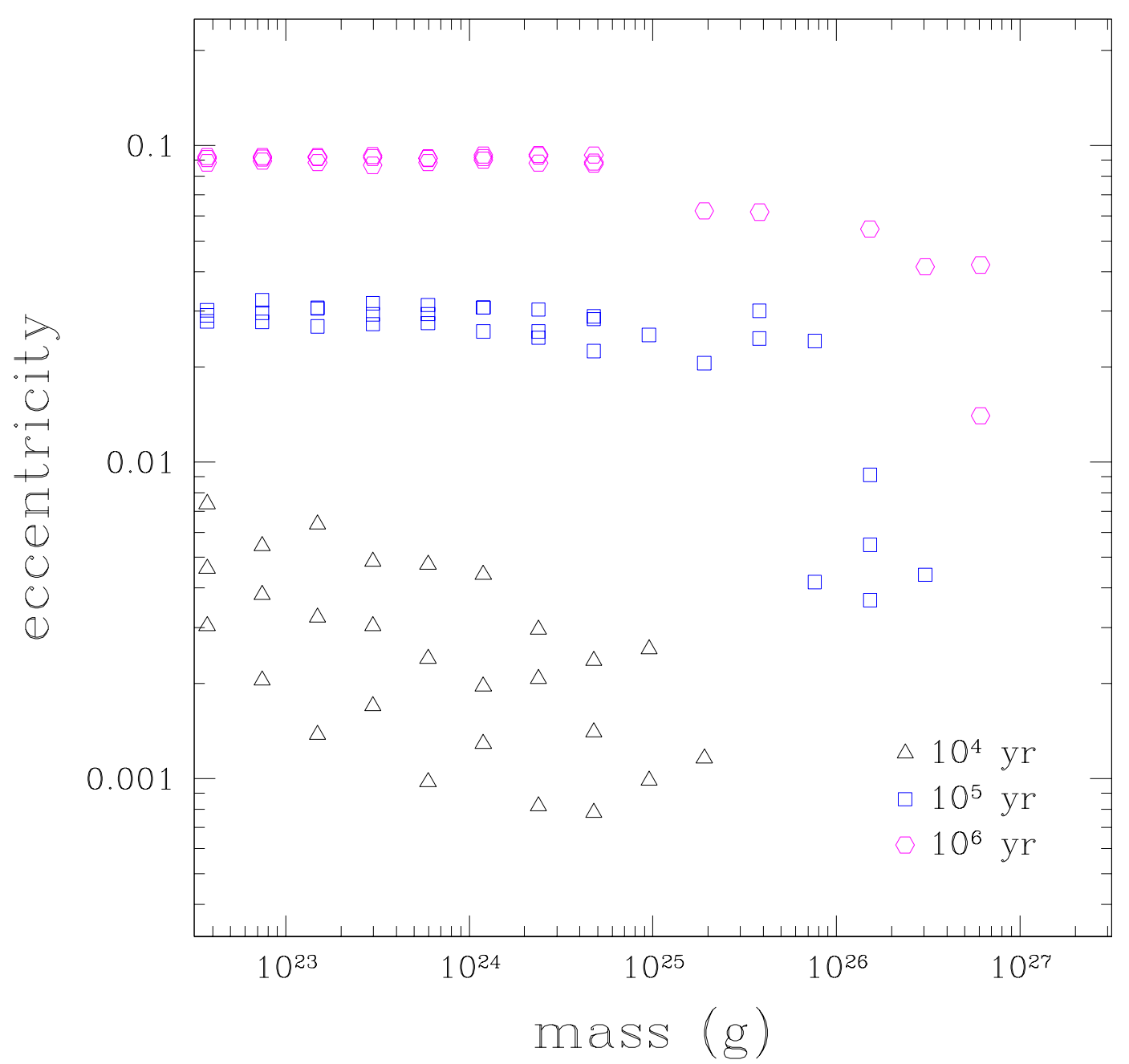

Fig. 9.- Evolution of eccentricity as a function of mass for hybrid calculations at 1 AU. The plots show the eccentricity at three different times, as indicated by the legend. For smaller mass values $\left(m<10^{25} \mathrm{~g}\right)$, averages are shown for three different annular regions within the computational grid. Typically, the eccentricities are higher for objects at smaller annuli, an effect which is more pronounced at earlier times. For larger mass values $\left(m>10^{25} \mathrm{~g}\right)$, the eccentricities of single objects are shown. These results illustrate the increase in viscous stirring of smaller mass objects with time, as well as the dynamical friction generally experienced by the most massive bodies. 


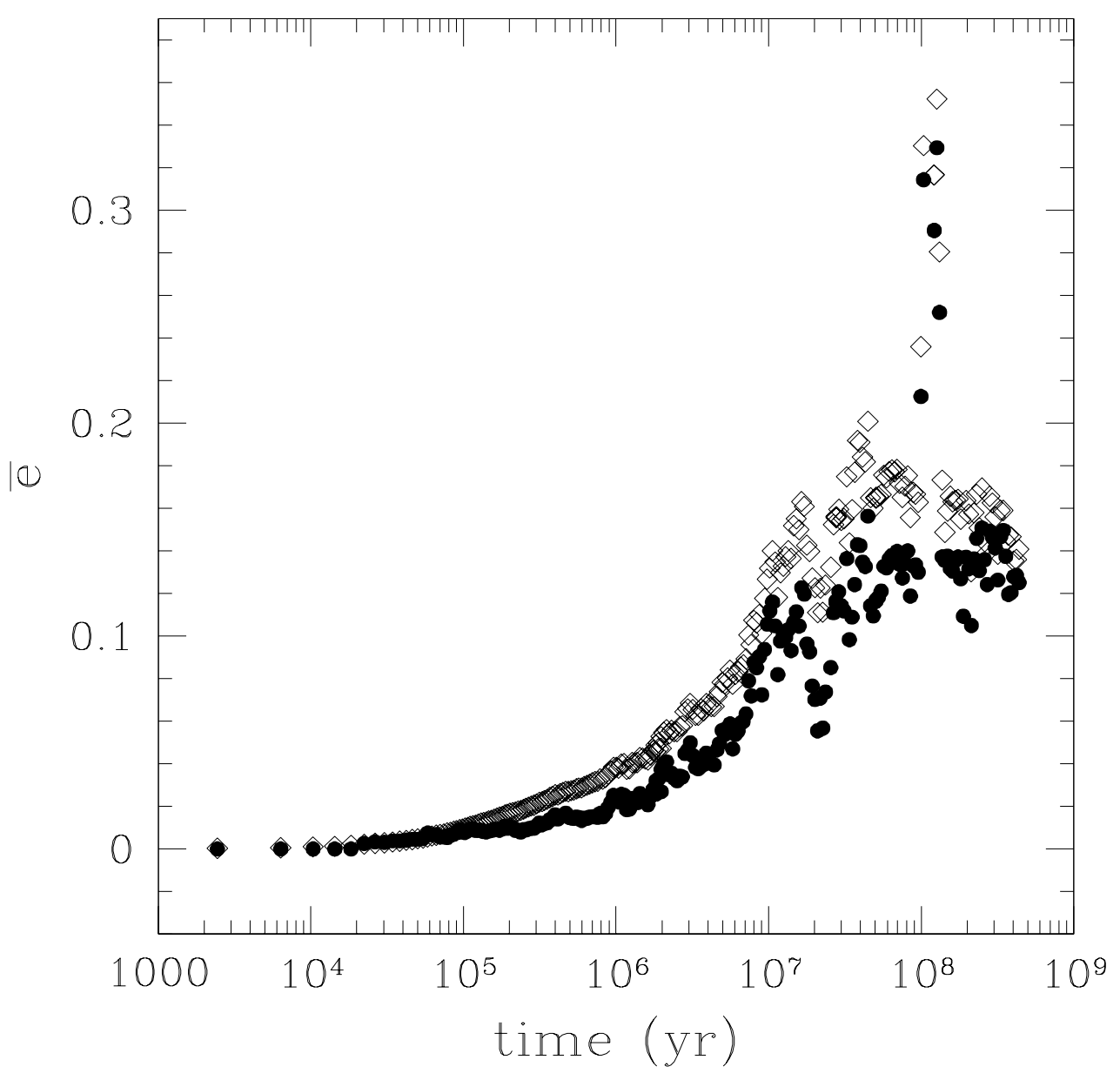

Fig. 10. - Evolution of the mass-weighted eccentricity $\bar{e}$ for a hybrid model. The solid circles show the evolution of the oligarchs; the open diamonds indicate the evolution of all objects in the grid. Viscous stirring and dynamical friction lead to a slow increase in $\bar{e}$. Strong dynamical interactions produce abrupt rises in $\bar{e}$; mergers produce abrupt declines. 


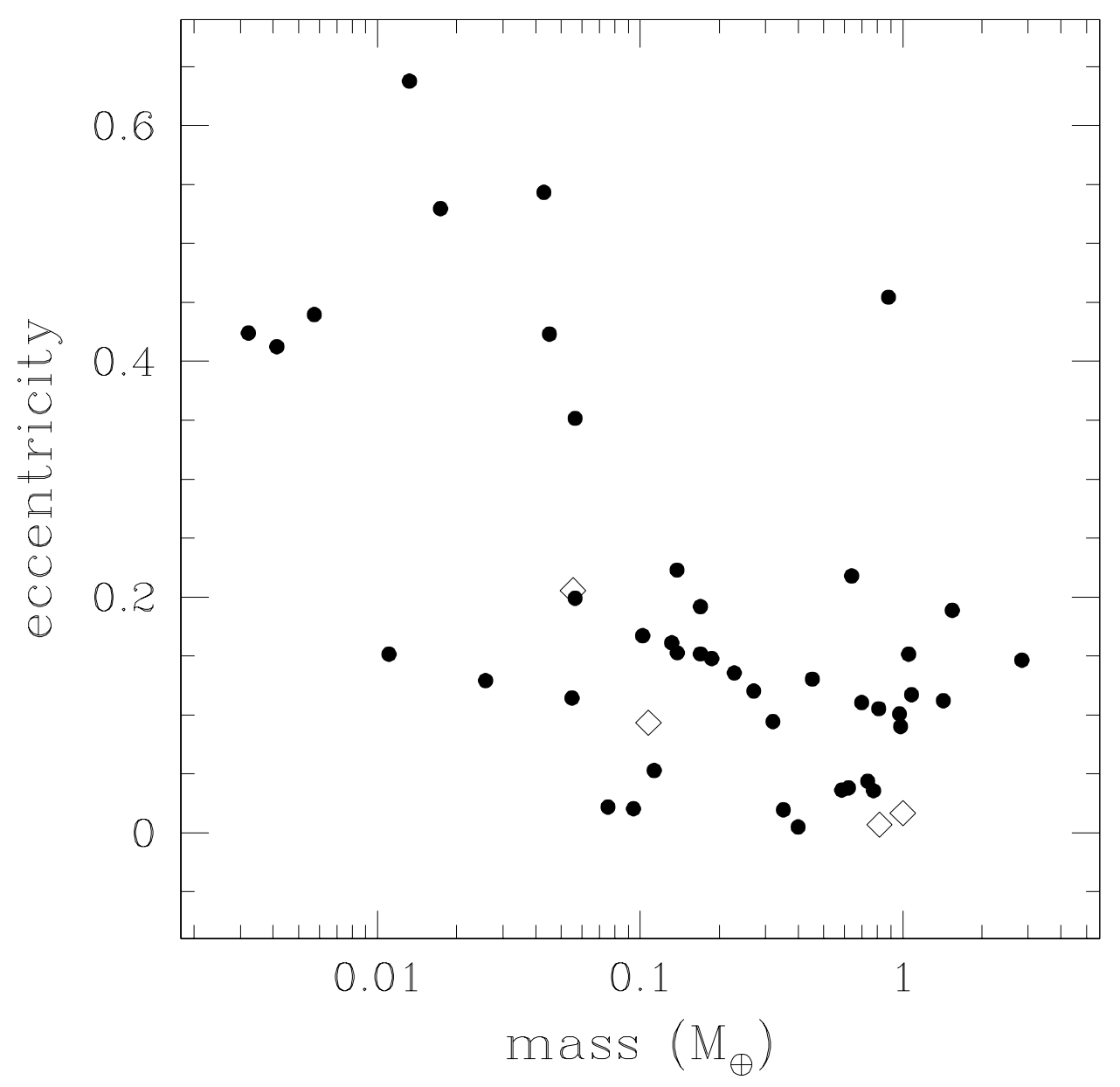

Fig. 11. - Final eccentricity as a function of final mass for calculations at $0.4-2$ AU. The open diamonds indicate current values for the terrestrial planets, Mercury, Venus, Earth, and Mars. The filled circles plot results for the ensemble of hybrid calculations listed in Table 1. 